Open Access

\title{
Outage probability analysis and optimal transmit power allocation for multi-hop full duplex relay network over Nakagami- $m$ fading channels
}

\author{
Rambabu Katla* (D) and A. V. Babu
}

\begin{abstract}
This paper investigates the outage probability and rate performance of multi-hop full duplex (FD) and half duplex (HD) decode-and-forward (DF)-based relay networks. First of all, we derive new closed form equation for the end-to-end outage probability of multi-hop FDR system taking into account the inter-relay interference (IRI), i.e., interference caused by simultaneous transmissions by the nodes in non-orthogonal frequency bands, and the residual self-interference (RSI) present at the full duplex relay (FDR) nodes, in independent non-identical Nakagami-m fading channels. We also derive an approximate expression for the outage probability which is found to be highly accurate. Further, we provide an asymptotic expression as well, for Rayleigh fading channels. Furthermore, we derive exact and approximate expression for the outage probability of multi-hop spectral efficient HDR network that employ two-phase relaying. We then consider optimal power allocation (OPA) for multi-hop FDR and HDR networks that maximizes the end-to-end transmission rate with individual peak power constraint at the nodes. Since the optimization problem is non-convex, we develop an efficient, low-complex and fast-converging iterative algorithm for power allocation based on sequential convex programming. Secondly, we consider OPA for multi-hop FDR and HDR networks that minimizes the end-to-end outage probability with individual peak power constraints at the nodes. We devise geometric programming (GP) to obtain the OPA vector. The results demonstrate that OPA can significantly improve the outage and end-to-end rate performance of both FDR and HDR networks as compared to uniform power allocation policy. The results from the analytical model are validated by extensive Monte Carlo simulations.
\end{abstract}

Keywords: Full duplex relaying, Decode-and-forward, Multi-hop transmission, Outage probability, Nakagami-m fading, Optimal power allocation

\section{Introduction}

In multi-hop wireless relay networks, communication between the source node and destination node is facilitated with the help of a number of relay nodes in cascade $[1,2]$. In conventional multi-hop relay networks, the relay nodes operate in half duplex mode. Recently, extensive investigations have been reported on the use of full duplex relaying (FDR) instead of half duplex relaying (HDR) [3]. The full duplex relay nodes are capable of performing reception and transmission concurrently using the same

*Correspondence: rambabu.katla@gmail.com

Department of Electronics and Communication Engineering, National Institute of Technology Calicut, 673601 Kozhikode, Kerala, India frequency band, resulting in improved spectral efficiency as compared to HDR systems. However, FDR systems suffer from self-interference (also known as echo or loop interference), arising due to imperfect isolation between the transmitting and receiving antennas of the relay, which can be mitigated by adopting effective antenna isolation and interference cancellation techniques. In spite of the advancements made in the design of such techniques, studies have shown that FDR systems are always affected by the presence of residual self-interference (RSI). On the other hand, HDR system does not suffer from RSI; however, the spectral efficiency is reduced significantly [3-6]. 
The end-to-end performance of multi-hop HDR networks has been studied extensively in the literature, e.g., $[1,2,7-11]$. Recently, the outage probability of a decodeand-forward (DF) FDR system was analyzed by a few researchers [4, 12-15]. In [4], the authors analyzed the outage probability of dual-hop FDR system assuming Rayleigh fading environment. This model was extended to a multi-hop FDR system in [12], while the authors of [13] have considered a dual-hop FDR system in Nakagami- $m$ fading channel. In $[14,15]$, the authors considered multihop FDR system in Nakagami- $m$ fading channels; however, closed-form equation for the outage probability was not provided. From the detailed survey of related work, it is observed that an exact closed-form analytical expression for the outage probability of multi-hop FDR system has not appeared in the literature so far considering Nakagami fading channels. Further, it is observed that there are no results available for asymptotic outage probability considering Rayleigh/Nakagami fading channels.

On the other hand, a few researchers have addressed the optimal power allocation (OPA) problem for FDR-based dual-hop and multi-hop networks [16-22]. In [16], the authors have proposed a hybrid full duplex/half duplex scheme for amplify-and-forward (AF) dual-hop network with transmit power adaption at the relay that maximizes the instantaneous and average spectral efficiency. In [17], OPA was derived for AF dual-hop FDR that maximizes the transmission rate of the network. In [18], OPA was derived under total and individual power constraints for dual-hop DF-based FDR system. Likewise, dual-hop FDR systems were considered in $[19,20]$ as well. In [21], OPA that maximizes the end-to-end throughput under a total power constraint was considered for a multi-hop FDR system while [22] considered OPA that minimizes the weighted sum of transmit powers under an outage probability constraint. However, work in [21] ignored the presence of RSI in the network while [22] assumed that both RSI and inter-relay interference (IRI) are negligible.

The first objective of this paper is to obtain closedform equation for the outage probability of a multi-hop DF-based FDR network by assuming the links to experience independent Nakagami- $m$ fading. We also derive an approximate expression for the outage probability which is more concise as compared to the exact expression. We validate the accuracy of the derived approximate expression through numerical and simulation studies. Further, we provide an asymptotic expression for the outage probability considering special case of Rayleigh fading channels. We then investigate OPA for multi-hop FDR system that maximizes the end-to-end instantaneous transmission rate with constraints on maximum transmit power at each node. We observe that the OPA problem is non-smooth and non-convex; accordingly, an efficient, low-complex, iterative power allocation algorithm based on sequential convex programming (SCP) has been devised to solve the OPA problem. Further, we provide the proof for the convergence of the proposed algorithm to a KarushKuhn-Tucker (KKT) point of the original problem, which guarantees the achievability of local optimal solution to the problem. We also make a comparison of the proposed algorithm with that of bisection-based power allocation algorithm and establish that SCP-based power allocation algorithm converges faster than the bisection-based algorithm. Furthermore, we consider OPA for multi-hop FDR system that minimizes the end-to-end outage probability with constraints on maximum transmit power at each node. We establish that the problem can be transformed to a standard geometric programming problem (GPP) and the OPA can be determined by solving the GPP. Moreover, we derive analytical equation for the outage probability of spectral efficient multi-hop HDR system described in [23] and then consider OPA for multi-hop HDR system as well. We describe the procedure to find the optimal transmit power for both FDR and HDR systems. Extensive simulation results are used to establish that the proposed power allocation algorithm converges very fast and is highly effective for solving the optimization problem considered in the paper. The results further demonstrate that OPA can significantly improve the end-to-end outage performance and transmission rate of both HDR and FDR systems as compared to uniform power allocation (UPA) policy.

Rest of the paper is organized as follows. Section 2 describes the system model. Theoretical derivation of the end-to-end outage probability of the multi-hop FDR/HDR systems are described in Section 3. Section 4 considers the OPA for FDR/HDR systems and describes the iterative algorithm to solve the optimization problem. The results are presented in Section 5 and the paper is concluded in Section 6.

\section{System model}

Multi-hop relaying is a promising technique to improve the coverage and throughput performance of wireless networks. In this case, intermediate relay nodes carry information from source node to destination node. Recently, this concept has been proposed for cooperative wireless networks [24] and cellular networks $[25,26]$. In relayassisted cellular networks, deployment of relay nodes can provide improved coverage for base stations and can enhance the signal quality experienced by cell edge user.

\subsection{Multi-hop FDR system}

A schematic diagram of the DF-based multi-hop FDR network is shown in Fig. 1a. Here, $F_{0}$ is the source node, $F_{N+1}$ is the destination node, and the relay nodes are represented as $F_{1}, F_{2} \ldots F_{N}$. Assume that $F_{0}$ transmits signal $x_{0}$ to the relay $F_{1}$ and let $y_{1}$ be the received signal at $F_{1}$. Since 

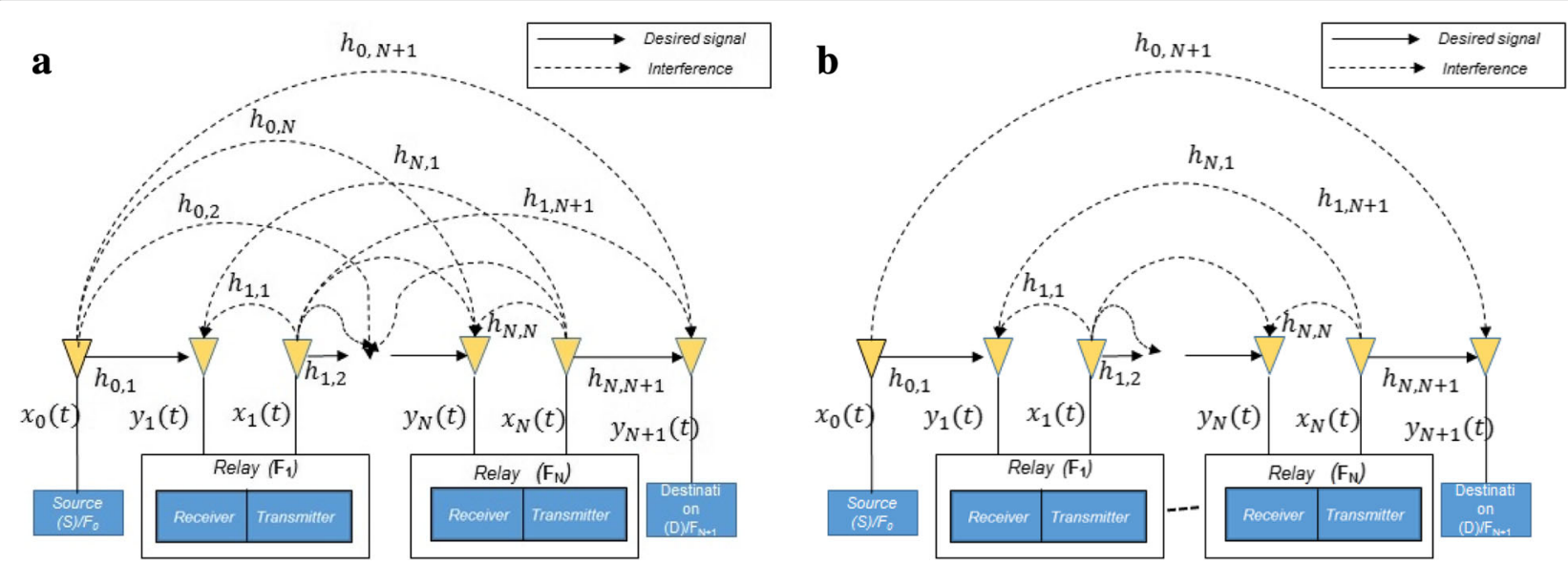

Fig. 1 Multi-hop system model. a FDR system. b Spectral efficient HDR system ( $N$ is even)

DF relaying is assumed, after decoding $y_{1}, F_{1}$ transmits $x_{1}$, which is a delayed version of $x_{0}$. This process is continued till the signal reaches $F_{N+1}$. Simultaneous transmission and reception by the FDR nodes in the same frequency band leads to self-interference at the relay nodes, which is caused by insufficient isolation between transmitter and receiver sections of the relay node. We assume the relay nodes to use two directional antennas, one for transmission and the other for reception. The directional antennas provide isolation between the transmission and reception for the self-interference channel, thereby reducing the self-interference.

Assume that $y_{j}$ and $x_{j}$ respectively represent the signals received and transmitted by $F_{j}$. Now, $y_{j}$ consists of the following: (i) actual information-bearing signal sent from $F_{j-1}$, (ii) RSI component present at $F_{j}$, and (iii) IRI from other relays $F_{i}, i=0,1, \ldots, N, i \neq\{j-1, j\}$ [27]. Let the channel gain between $F_{i}$ and $F_{j}$ be $h_{i, j},\{i, j \in(0,1 \ldots . N+1)\}$. We consider block fading channel where the channel gains remain constant in each transmission block (i.e., each block contains $n$ number of symbols) and change independently in the next block. We assume $h_{i, j}$ to follow Nakagami- $m$ distribution with shape parameter $m_{i, j}$ and mean power $\mathbf{E}\left[\left|h_{i, j}\right|^{2}\right]=\pi_{i, j}$. Accordingly, $\left|h_{i, j}\right|^{2}$ is a Gamma random variable with shape parameter $m_{i, j}$ and scale parameter $\frac{\pi_{i, j}}{m_{i, j}}$. The probability distribution function (PDF) of $\left|h_{i, j}\right|$ is given by [28]

$$
f_{\left|h_{i, j}\right|}(x)=\left(\frac{m_{i, j}}{\pi_{i, j}}\right)^{m_{i, j}} \frac{2 x^{\left(2 m_{i, j}-1\right)}}{\Gamma\left(m_{i, j}\right)} e^{-\left(\frac{m_{i, j} x^{2}}{\pi_{i, j}}\right)}
$$

where $\Gamma($.$) is the Gamma function. As reported in$ $[4,5,12-20]$, we consider that the RSI channel at relay $F_{j}$, (after employing all known practical interference isolation and cancellation techniques), can be modeled as a fading channel. Let $h_{j, j}$ be the fading coefficient corresponding to the RSI channel at $F_{j}$. We assume $h_{j, j}(j=0,1, . . N)$ to follow Nakagami- $m$ fading model, since it can span a wide range of fading scenarios that include both Rayleigh as well as Rician.

The equation for the received signal $y_{j}$ at $F_{j}$ for a given time instant is given by:

$$
y_{j}=h_{j-1, j} x_{j-1}+h_{j, j} x_{j}+\sum_{\substack{i=0, i \neq j \\ i \neq j-1}}^{N} h_{i, j} x_{i}+n_{j}
$$

where $n_{j}$ is the additive white Gaussian noise component at $F_{j}$ with power $\sigma_{j}^{2}$. Notice that (2) considers the signals that arrive at $F_{j}$ from $F_{i}\{i \neq j-1\}$, as interference, i.e., it is assumed that $F_{j}$ does not implement any sort of diversity techniques as reported in [29].

\subsection{Multi-hop HDR system}

The conventional $K$-hop HDR systems require $K$ orthogonal time slots for communication which degrades the spectral efficiency. For fair comparison of FDR network against equivalent HDR network, the spectral efficient HDR system proposed in [23] is considered, which uses two-phase relaying and is illustrated in Fig $1 \mathrm{~b}$. Here, nodes $F_{0}, F_{2}, F_{4} \ldots$ transmit in the first time slot while all other nodes are in the receive mode. In the second time slot, nodes $F_{1}, F_{3}, F_{5} \ldots$.., transmit while the rest of the nodes stay in the receive mode.

Let $y_{j}^{0}$ and $x_{j}^{0}$ respectively be the received and transmitted signals at a given time instance at node $F_{j}$. Now, $y_{j}^{0}$ consists of two components: (i) actual information bearing signal sent from $F_{j-1}$ and (ii) IRI from other relays $F_{p}$, where $p=2 i+q, i=0,1, \ldots\left\lfloor\frac{N-q}{2}\right\rfloor$ and $q$ represents the remainder when $j-1$ is divided by 2 . Accordingly, the received signal $y_{j}^{0}$ at $F_{j}$ can be written as: 


$$
y_{j}^{0}=h_{j-1, j} x_{j-1}^{0}+\sum_{\substack{i=0 \\ p \neq j-1}}^{\left\lfloor\frac{N-q}{2}\right\rfloor} h_{p, j} x_{p}^{0}+n_{j}
$$

In Section 3.2, we investigate the outage probability and rate performance of multi-hop spectral efficient HDR network and make an extensive comparison study among multi-hop FDR/HDR systems. Even though the spectral efficient HDR has been proposed in [23], the optimal power allocation, outage, and rate performance were not evaluated in the literature so far.

\section{Derivation of outage probability}

This section describes the analytical model for finding the outage probability of multi-hop FDR/HDR sytem considering Nakagmi- $m$ fading scenario.

\subsection{Outage probability of multi-hop FDR system}

Let $\mathbf{P}=\left(P_{0}, P_{1}, \ldots, P_{N}\right)^{T}$ be the transmit power vector, where $P_{i}$ represents the transmission power of relay node $F_{i}$. Let $\Gamma_{j}(\mathbf{P})$ be the signal-to-interference-plus-noise ratio at $F_{j} ; j=1, \ldots, N+1$. Now, $\Gamma_{j}(\mathbf{P})$ is given by

$$
\Gamma_{j}(\mathbf{P})=\frac{P_{j-1}\left|h_{j-1, j}\right|^{2}}{\sigma_{j}^{2}+\sum_{\substack{i=0 \\ i \neq j-1}}^{N} P_{i}\left|h_{i, j}\right|^{2}}
$$

The interference power terms in (4) include both RSI as well as IRI. Let us define $X_{i, j}=\frac{P_{i}\left|h_{i, j}\right|^{2}}{\sigma_{j}^{2}},\{i, j \in(0,1 \ldots . N+1)\}$. Since the channel coefficients $\left|h_{i, j}\right|^{2}$ have Gamma PDF, the random variables $\left\{X_{i, j}\right\}$ also have Gamma PDF with shape parameters $m_{i, j}$ and scale parameters $\beta_{i, j}$. The PDF of $X_{i, j}$ can be determined using the results of random variable transformation and is given by [28]:

$$
f_{X_{i, j}}(x)=\beta_{i, j}^{-m_{i, j}} \frac{x^{m_{i, j}-1}}{\Gamma\left(m_{i, j}\right)} e^{-\left(\frac{x}{\beta_{i, j}}\right)}
$$

where $\beta_{i, j}=\frac{\pi_{i, j}}{m_{i, j}} \frac{P_{i}}{\sigma_{j}^{2}}$. Now, (4) can be written as follows:

$$
\Gamma_{j}(\boldsymbol{P})=\frac{X_{j-1, j}}{1+\sum_{\substack{i=0 \\ i \neq j-1}}^{N} X_{i, j}}
$$

Assuming complex Gaussian inputs, unit bandwidth, and that the nodes adopt a continuous rate scheme, the instantaneous transmission rate of the link between $F_{j-1}$ and $F_{j}$ is given by:

$$
R_{j}(\boldsymbol{P})=\log \left(1+\Gamma_{j}(\boldsymbol{P})\right)
$$

In addition, the end-to-end instantaneous rate of the source to destination link for DF relaying is the minimum of the instantaneous transmission rates of the $N+1$ links and is given by [27]

$$
R(\boldsymbol{P})=\min _{j}\left(R_{j}(\boldsymbol{P})\right), \quad j=1,2, \ldots, N+1
$$

The end-to-end outage probability of multi-hop DF FDR system is defined as the probability that end-to-end instantaneous rate is less than the given target rate $r$ and is given by

$$
\begin{aligned}
P_{\text {out }}^{F D R} & =\operatorname{Pr}\left(\min _{j}\left(R_{j}(\boldsymbol{P})\right)<r\right) \\
& =1-\prod_{j=1}^{N+1} \operatorname{Pr}\left(R_{j}(\boldsymbol{P}) \geq r\right) \\
& =1-\prod_{j=1}^{N+1} \operatorname{Pr}\left(\Gamma_{j}(\boldsymbol{P}) \geq \Gamma_{T}\right)
\end{aligned}
$$

where $\operatorname{Pr}($.$) denotes probability and \Gamma_{T}$ is the SINR threshold, which is related to the link target transmission rate, $r$, through the relation $\Gamma_{T}=2^{r}-1$ [4]. The $j$ th term in (9) is given by

$$
\operatorname{Pr}\left(\Gamma_{j}(\boldsymbol{P}) \geq \Gamma_{T}\right)=\operatorname{Pr}\left(\frac{X_{j-1, j}}{1+\sum_{\substack{i=0 \\ i \neq j-1}}^{N} X_{i, j}} \geq \Gamma_{T}\right)
$$

Let $Y_{j}=\sum_{\substack{i=0 \\ i \neq j-1}}^{N} X_{i, j}$ and $W_{j}=\frac{X_{j-1, j}}{\Gamma_{T}}$. Further, let $f_{Y_{j}}(y)$ and $f_{W_{j}}(w)$ respectively be the PDFs of $Y_{j}$ and $W_{j}$. Now, (10) becomes

$$
\begin{aligned}
\operatorname{Pr}\left(\Gamma_{j}(\boldsymbol{P}) \geq \Gamma_{T}\right) & =\operatorname{Pr}\left(W_{j} \geq 1+Y_{j}\right) \\
& =\int_{0}^{\infty} f_{Y_{j}}(y) \int_{y+1}^{\infty} f_{W_{j}}(w) d w d y
\end{aligned}
$$

Using (5), the PDF of $W_{j}$ can be written as follows:

$$
f_{W_{j}}(w)=\left(\frac{\beta_{j-1, j}}{\Gamma_{T}}\right)^{-m_{j-1, j}} \frac{w^{m_{j-1, j}-1}}{\Gamma\left(m_{j-1, j}\right)} e^{-\left(\frac{w \Gamma_{T}}{\beta_{j-1, j}}\right)}
$$

\subsubsection{Exact expression for outage probability}

Here, we derive the exact expression for the outage probability of multi-hop FDR network. Since $Y_{j}$ is the sum of independent Gamma random variables, for integer values of Nakagami parameter $m_{i, j}(i \neq j-1, i=0 \ldots . N)$, the exact PDF of $Y_{j}$, i.e., $f_{Y_{j}}(y)$ is given by [30]:

$$
f_{Y_{j}}(y)=\sum_{\substack{i=0 \\ i \neq j-1}}^{N} \sum_{k=1}^{m_{i, j}} \Xi\left(i, k,\left\{m_{i, j}\right\},\left\{\beta_{i, j}\right\}\right) f_{X_{i, j}}(y)
$$

where the weights $\Xi\left(i, k,\left\{m_{i, j}\right\},\left\{\beta_{i, j}\right\}\right)$ can be found using the recursive formula given by [30]:

$$
\begin{aligned}
\Xi\left(i, m_{i, j}-k\right)= & \frac{1}{k} \sum_{l=1}^{k} \sum_{\substack{q=0 \\
q \neq i}}^{N} \frac{m_{q, j}}{\beta_{i, j}^{l}}\left(\frac{1}{\beta_{i, j}}-\frac{1}{\beta_{q, j}}\right)^{-l} \\
& \times \Xi\left(i, m_{i, j}-k+l\right)
\end{aligned}
$$




$$
\Xi\left(i, m_{i, j}\right)=\frac{\beta_{i, j}^{m_{i, j}}}{\prod_{h=0}^{N} \beta_{h, j}^{m_{h, j}}} \prod_{\substack{l=0 \\ l \neq i}}^{N}\left(\frac{1}{\beta_{l, j}}-\frac{1}{\beta_{i, j}}\right)^{-m_{l, j}}
$$

Substituting (13) in (11) and interchanging the order of summation and integration, $\operatorname{Pr}\left(\Gamma_{j}(\boldsymbol{P}) \geq \Gamma_{T}\right)$ can be written as follows:

$$
\begin{aligned}
\operatorname{Pr}\left(\Gamma_{j}(\boldsymbol{P}) \geq \Gamma_{T}\right)= & \sum_{\substack{i=0 \\
i \neq j-1}}^{N} \sum_{k=1}^{m_{i, j}} \Xi(i, k) \int_{0}^{\infty} f_{X_{i, j}}(y) \\
& \times \int_{y+1}^{\infty} f_{W_{j}}(w) d w d y
\end{aligned}
$$

Now, substituting (5) and (12) in (16) and after some mathematical simplifications, (16) yields the following:

$$
\begin{aligned}
\operatorname{Pr}\left(\Gamma_{j}(\boldsymbol{P}) \geq \Gamma_{T}\right)= & \sum_{\substack{i=0 \\
i \neq j-1}}^{N} \sum_{k=1}^{m_{i, j}} \frac{\Xi(i, k)\left(\frac{\Gamma_{T}}{\beta_{j-1, j}}\right)^{m_{j-1, j}}}{\beta_{i, j}^{m_{i, j}} \Gamma\left(m_{i, j}\right) \Gamma\left(m_{j-1, j}\right)} \\
& \times \int_{0}^{\infty} y^{m_{i, j}-1} e^{-\frac{y}{\beta_{i, j}}} \int_{y+1}^{\infty} w^{m_{j-1, j}-1} e^{-\frac{w \Gamma_{T}}{\beta_{j-1, j}}} d w d y
\end{aligned}
$$

The final equation for the outage probability is obtained by solving (17) and substituting the result in (9) and is given by (refer to Appendix 1 for the detailed derivation):

$$
\begin{aligned}
P_{\text {out }}^{F D R}= & -\prod_{j=1}^{N+1} \sum_{\substack{i=0 \\
i \neq j-1}}^{N} \sum_{k=1}^{m_{i, j}} \sum_{n=0}^{m_{j-1, j}-1} \sum_{r=0}^{n} \frac{\Xi(i, k)}{n !} e^{-\left(\frac{\Gamma_{T}}{\beta_{j-1, j}}\right)} \\
& \times\left(\begin{array}{l}
n \\
r
\end{array}\right) \beta_{i, j}^{r}\left(\frac{\Gamma_{T}}{\beta_{j-1, j}}\right)^{n}\left(1+\frac{\Gamma_{T} \beta_{i, j}}{\beta_{j-1, j}}\right)^{-\left(m_{i, j}+r\right)} \frac{\Gamma\left(m_{i, j}+r\right)}{\Gamma\left(m_{i, j}\right)}
\end{aligned}
$$

\subsubsection{Approximate expression for outage probability}

Since the exact outage probability given by (18) contains weights $\Xi(i, k)$ which are to be computed recursively, outage probability calculation is computationally expensive. To reduce the computational complexity, we derive an approximate simplified expression based on WelchSatterwaite approximation for the sum of Gamma random variables [31]. In Section 5 of this paper, we validate the accuracy of the approximate expression through numerical illustrations. Notice that finding the outage probability expression requires $f_{Y_{j}}(y)$, which is the PDF of the sum of $N$ independent Gamma random variables. According to [31], sum of $N$ independent Gamma-distributed random variables can be approximated by a Gamma random variable. Accordingly, the PDF of the approximated Gamma random variable $\tilde{Y}_{j}, f_{\tilde{Y}_{j}}(y)$ can be written as follows:

$$
f_{\tilde{Y}_{j}}(y)=\beta_{j}^{-m_{j}} \frac{y^{m_{j}-1}}{\Gamma\left(m_{j}\right)} e^{-\left(\frac{y}{\beta_{j}}\right)}
$$

where $m_{j}=\frac{\left(\sum_{\substack{i=0 \\ i \neq j-1}}^{N} m_{i, j} \beta_{i, j}\right)^{2}}{\sum_{\substack{i=0 \\ i \neq j-1}}^{N} m_{i, j} \beta_{i, j}^{2}}$ and $\beta_{j}=\frac{\sum_{\substack{i \neq 0 \\ i \neq j-1}}^{N} m_{i, j} \beta_{i, j}^{2}}{\sum_{\substack{i=0 \\ i \neq j-1}}^{N} m_{i, j} \beta_{i, j}}$ are the shape and scale parameters of $\tilde{Y}_{j}$. An approximate expression for the probability $\operatorname{Pr}\left(\Gamma_{j} \geq \Gamma_{T}\right)$ can be determined by substituting $f_{W_{j}}(w)$ given by $(12)$ and $f_{\tilde{Y}_{j}}(y)$ given by (19) in (11), i.e., $f_{\tilde{Y}_{j}}(y)$ is used instead of $f_{Y_{j}}(y)$ in (11). After rearranging the relevant terms, the following expression can be obtained for this probability, i.e.,

$$
\begin{aligned}
\tilde{\operatorname{Pr}}\left(\Gamma_{j} \geq \Gamma_{T}\right)= & \frac{\beta_{j}^{-m_{j}}}{\Gamma\left(m_{j}\right) \Gamma\left(m_{j-1, j}\right)}\left(\frac{\beta_{j-1, j}}{\Gamma_{T}}\right)^{-m_{j-1, j}} \\
& \times \int_{0}^{\infty} y^{m_{j}-1} e^{-\left(\frac{y}{\beta_{j}}\right)} \int_{y+1}^{\infty} w^{m_{j-1, j}-1} e^{-\left(\frac{w \Gamma_{T}}{\beta_{j-1, j}}\right)} d w d y
\end{aligned}
$$

Notice that the double integral term in (20) is similar to that given in (17). This integral can be solved using an approach similar to the one followed for the derivation of (18), described in Appendix 1. By following the steps laid out in Appendix 1, the following expression can be obtained:

$$
\begin{aligned}
\tilde{\operatorname{Pr}}\left(\Gamma_{j} \geq \Gamma_{T}\right)= & \sum_{n=0}^{m_{j-1, j}-1} \sum_{r=0}^{n} e^{-\left(\frac{\Gamma_{T}}{\beta_{j-1, j}}\right)} \frac{\left(\frac{\Gamma_{T}}{\beta_{j-1, j}}\right)^{n}}{n !} \\
& \times\left(\begin{array}{l}
n \\
r
\end{array}\right) \beta_{j}^{r}\left(1+\frac{\Gamma_{T} \beta_{j}}{\beta_{j-1, j}}\right)^{-\left(m_{j}+r\right)} \frac{\Gamma\left(m_{j}+r\right)}{\Gamma\left(m_{j}\right)}
\end{aligned}
$$

Now, substituting (21) in (9), we get the approximate closed-form expression for the outage probability of multihop FDR system as follows:

$$
\begin{aligned}
\tilde{P}_{\text {out }}^{F D R}= & -\prod_{j=1}^{N+1} \sum_{n=0}^{m_{j-1, j}-1} \sum_{r=0}^{n} e^{-\left(\frac{\Gamma_{T}}{\beta_{j-1, j}}\right)} \frac{\left(\frac{\Gamma_{T}}{\beta_{j-1, j}}\right)^{n}}{n !} \\
& \times\left(\begin{array}{l}
n \\
r
\end{array}\right) \beta_{j}^{r}\left(1+\frac{\Gamma_{T} \beta_{j}}{\beta_{j-1, j}}\right)^{-\left(m_{j}+r\right)} \frac{\Gamma\left(m_{j}+r\right)}{\Gamma\left(m_{j}\right)}
\end{aligned}
$$

The simplified outage probability expression for the special case of Rayleigh fading can be obtained by substituting $m_{i, j}=1 \forall i, j$ in (22) and is given as follows:

$$
\hat{P}_{\text {out }}^{F D R}=1-\prod_{j=1}^{N+1} e^{-\left(\frac{\Gamma_{T}}{\hat{\beta}_{j-1, j}}\right)}\left(1+\frac{\Gamma_{T} \hat{\beta}_{j}}{\hat{\beta}_{j-1, j}}\right)^{-\hat{m}_{j}}
$$


where $\hat{m}_{j}=\frac{\left(\sum_{\substack{i=0 \\ i \neq j-1}}^{N} \hat{\beta}_{i, j}\right)^{2}}{\sum_{\substack{i=0 \\ i \neq j-1}}^{N} \hat{\beta}_{i, j}^{2}}, \hat{\beta}_{j}=\frac{\sum_{\substack{i=0 \\ i \neq j-1}}^{N} \hat{\beta}_{i, j}^{2}}{\sum_{\substack{i=0 \\ i \neq j-1}}^{N} \hat{\beta}_{i, j}}$ and $\hat{\beta}_{i, j}=$ $\pi_{i, j}\left(\frac{P_{i}}{\sigma_{j}^{2}}\right)$

The amount of RSI power at a FDR node is directly proportional to the transmit power at the relay node. In the high transmit power region, the amount of RSI and IRI would be very high. This leads to an outage probability floor for FDR networks in the high transmit power region, i.e., outage probability remains constant as the transmit power increases. An expression for asymptotic outage probability would be useful to theoretically verify the existence of outage floor behavior in muti-hop FDR networks. Recall that Rayleigh fading, which is applicable for a rich multi-path environment, is a special case of Nakagami fading [28]. So asymptotic outage results for Rayleigh fading are relevant under certain channel conditions. Accordingly, we derive the asymptotic outage probability of multi-hop FDR system under Rayleigh fading, which can be obtained by letting $P_{i} \rightarrow \infty$ in (23). This implies $\hat{\beta}_{i, j} \rightarrow \infty$ and $\hat{\beta}_{j} / \hat{\beta}_{j-1, j}<<1$. By using the binomial linear approximation $(1+x)^{-n} \approx 1-n x \approx \exp (-n x)$ for small $x$ and substituting $\hat{\beta}_{j}=\left(\sum_{\substack{i=0 \\ i \neq j-1}}^{N} \hat{\beta}_{i, j}\right) / \hat{m}_{j}$ in (23), the asymptotic outage probability is given as follows:

$$
\hat{P}_{\text {out }, a s y}^{F D R}=1-\prod_{j=1}^{N+1} \exp \left[-\frac{\Gamma_{T}}{\hat{\beta}_{j-1, j}}\left(1+\sum_{\substack{i=0 \\ i \neq j-1}}^{N} \hat{\beta}_{i, j}\right)\right]
$$

Now, (24) can be used to describe the outage probability floor behavior which occurs when the links are unbalanced, i.e., when $\hat{\beta}_{b-1, b}<<\hat{\beta}_{j-1, j} \forall j, j \neq b$, the product of exponentials terms in (24) becomes very low due to the fact that the desired signal over the $b$ th link becomes very poor and it cannot be improved by increasing the transmit power, which leads to outage floor behavior. Similarly, when the interference at a node is very high as compared to the other nodes, it also leads to outage floor. However, the product term in (24) takes the higher possible numerical value when all the links are balanced and it provides minimum value for the outage probability. Since $\hat{\beta}_{i, j}$ depends on the transmit powers of the nodes, optimal allocation of power among the nodes results in balanced links which leads to improved outage probability performance.

\subsection{Outage probability of multi-hop HDR system}

For the spectral efficient multi-hop HDR system described in [23], the SINR at relay node $F_{j}, \Gamma_{j}^{H D R}(\boldsymbol{P})$ is given by

$$
\Gamma_{j}^{H D R}(\boldsymbol{P})=\frac{P_{j-1}\left|h_{j-1, j}\right|^{2}}{\sigma_{j}^{2}+\sum_{\substack{\left\lfloor\frac{N-q}{i=0} \\ p \neq j-1\right.}}^{2} P_{p}\left|h_{p, j}\right|^{2}}
$$

The achievable end-to-end instantaneous rate of the multi-hop HDR system is the minimum of the achievable instantaneous rate over each link [27] and is given by

$$
R^{H D R}(\boldsymbol{P})=\min _{j}\left(R_{j}^{H D R}(\boldsymbol{P})\right), \quad j=1,2, \ldots, N+1
$$

where $R_{j}^{H D R}(\boldsymbol{P})$ is the achievable instantaneous rate of link $j, j=1,2, \ldots, N+1$ and is given as follows:

$$
R_{j}^{H D R}(\boldsymbol{P})=\frac{1}{2} \log \left(1+\Gamma_{j}^{H D R}(\boldsymbol{P})\right)
$$

The fraction $1 / 2$ in (27) is due to the fact that spectral efficient multi-hop HDR requires two orthogonal time slots for transmission and reception $[4,12]$.

The end-to-end outage probability of multi-hop DF HDR system is defined as the probability that end-to-end instantaneous rate is less than the given target rate $r$ and is given by

$$
\begin{aligned}
P_{\text {out }}^{H D R} & =\operatorname{Pr}\left(\min _{j}\left(R_{j}^{H D R}(\boldsymbol{P})\right)<r\right) \\
& =1-\prod_{j=1}^{N+1} \operatorname{Pr}\left(R_{j}^{H D R}(\boldsymbol{P}) \geq r\right) \\
& =1-\prod_{j=1}^{N+1} \operatorname{Pr}\left(\Gamma_{j}^{H D R}(\boldsymbol{P}) \geq \gamma_{T}\right)
\end{aligned}
$$

Here, the threshold SINR $\left(\gamma_{T}\right)$ for HDR systems is given as $\gamma_{T}=2^{2 r}-1$, where $r$ is the target rate of the FDR system. Notice that (28) is similar to (9) with $\Gamma_{j}^{H D R}(\boldsymbol{P})$ given by (25). By adopting an approach similar to multihop FDR systems, it can be shown that the exact outage probability of the spectral efficient multi-hop HDR system in the presence of independent nonidentical Nakagami- $m$ fading channels is given by the following equation:

$$
\begin{aligned}
P_{\text {out }}^{H D R}= & -\prod_{j=1}^{N+1} \sum_{\substack{i=0 \\
p \neq j-1}}^{\left\lfloor\frac{N-q}{2}\right\rfloor} \sum_{k=1}^{m_{p, j}} \sum_{n=0}^{m_{j-1, j-1}-1} \sum_{r=0}^{n} \frac{\Xi(p, k)}{n !} e^{-\left(\frac{\gamma_{T}}{\beta_{j-1, j}}\right)} \\
& \times\left(\begin{array}{l}
n \\
r
\end{array}\right) \beta_{p, j}^{r}\left(\frac{\gamma_{T}}{\beta_{j-1, j}}\right)^{n}\left(1+\frac{\gamma_{T} \beta_{p, j}}{\beta_{j-1, j}}\right)^{-\left(m_{p, j}+r\right)} \frac{\Gamma\left(m_{p, j}+r\right)}{\Gamma\left(m_{p, j}\right)}
\end{aligned}
$$

where the coefficients $\Xi(p, k)$ are computed recursively as given by (14) and (15). To get a simplified expression for the outage probability, we use the Welch-Satterwaite approximation for the sum of $N$ independent Gamma random variables [31] as used in Section III-A for the multi-hop FDR system. By adopting a similar approach, the following approximate expression can be obtained 
for the outage probability of multi-hop HDR system in independent nonidentical Nakagami- $m$ fading channels:

$$
\begin{aligned}
\tilde{P}_{\text {out }}^{H D R}= & -\prod_{j=1}^{N+1} \sum_{n=0}^{m_{j-1, j}-1} \sum_{r=0}^{n} e^{-\left(\frac{\gamma_{T}}{\beta_{j-1, j}}\right)} \frac{\left(\frac{\gamma_{T}}{\beta_{j-1, j}}\right)^{n}}{n !}\left(\begin{array}{l}
n \\
r
\end{array}\right) \\
& \times \tilde{\beta}_{j}^{r}\left(1+\frac{\gamma_{T} \tilde{\beta}_{j}}{\beta_{j-1, j}}\right)^{-\left(\tilde{m}_{j}+r\right)} \frac{\Gamma\left(\tilde{m}_{j}+r\right)}{\Gamma\left(\tilde{m}_{j}\right)}
\end{aligned}
$$

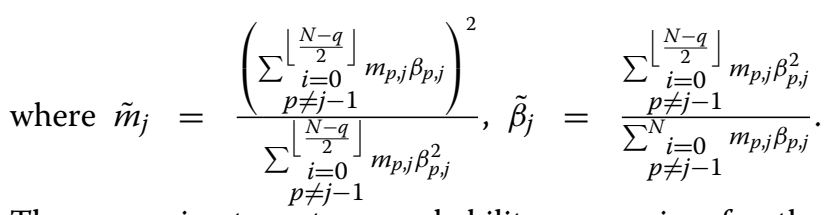
The approximate outage probability expression for the Rayleigh fading case is obtained by substituting $m_{i, j}=1 \forall i, j$ in (30) and is given by

$$
\bar{P}_{\text {out }}^{H D R}=1-\prod_{j=1}^{N+1} e^{-\left(\frac{\gamma_{T}}{\bar{\beta}_{j-1, j}}\right)}\left(1+\frac{\gamma_{T} \bar{\beta}_{j}}{\bar{\beta}_{j-1, j}}\right)^{-\bar{m}_{j}}
$$

Similar to (24), the asymptotic outage probability of multihop HDR system for the Rayleigh fading case can be obtained as follows:

$$
\bar{P}_{\text {out }, \text { asy }}^{H D R}=1-\prod_{j=1}^{N+1} \exp \left[-\frac{\gamma_{T}}{\bar{\beta}_{j-1, j}}\left(\sum_{\substack{i=0 \\ p \neq j-1}}^{\left\lfloor\frac{N-q}{2}\right\rfloor} \bar{\beta}_{p, j}\right)\right]
$$

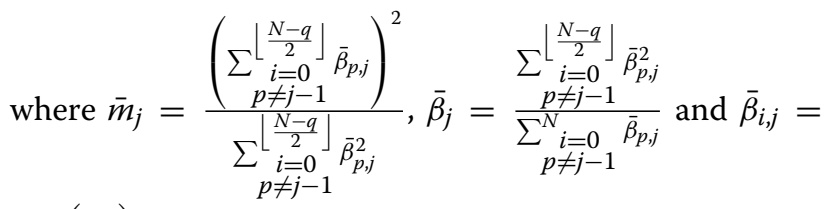
$\pi_{i, j}\left(\frac{P_{i}}{\sigma_{j}^{2}}\right)$

\section{Optimal power allocation}

In this section, we address two power allocation problems that optimize the performance of multi-hop FDR and HDR systems. The first problem investigates the maximization of end-to-end rate, while the second problem investigates the minimization of end-to-end outage probability of multi-hop FDR and HDR systems, with constraints on maximum transmit power at the nodes. Note that rate maximization requires the knowledge of complete instantaneous channel state information (I-CSI) at the nodes and outage probability minimization requires the availability of statistical channel state information (S-CSI) alone.

\subsection{Optimal power allocation for multi-hop FDR system} Here, we formulate two OPA problems for multi-hop FDR system: (i) for maximizing the end-to-end instantaneous rate and (ii) for minimizing the end-to-end outage probability. We establish that the rate maximization problem is non-convex. We then propose an iterative power allocation algorithm based on sequential convex programming (SCP) for finding the OPA vector that meets the objective. On the other hand, we transform the outage probability minimization into a standard geometric programming problem (GPP) and establish that the optimal solution can be obtained by solving the GPP.

\subsubsection{End-to-end instantaneous rate maximization}

Here, we formulate the OPA problem that maximizes the end-to-end instantaneous rate of the system. Since the objective function (i.e., rate) is non-concave, we show that the optimization problem is non-convex and derive the optimality conditions for the problem. We then propose an iterative power allocation algorithm based on SCP for finding the OPA vector that meets the objective.

The objective of the OPA problem is to maximize the end-to-end instantaneous rate subject to the constraint on maximum transmit power $\left(P_{i, \max }\right)$ at each node $F_{i}$. This problem can be formulated as

$$
\begin{aligned}
& \max _{\boldsymbol{P}} \min _{j}\left\{R_{j}(\boldsymbol{P})=\log \left(1+\Gamma_{j}(\boldsymbol{P})\right)\right\} \\
& \text { subject to } \quad 0 \leq P_{j-1} \leq P_{j-1, \text { max }} \forall j .
\end{aligned}
$$

Since the objective function in (33a) is a non-smooth and non-concave function of $\boldsymbol{P}$ [32], the optimization problem (33) is non-convex and it is not possible to find the global optimal solution. However, the non-smooth optimization problem can be converted to a smooth optimization problem and the optimality condition can be derived by writing the hypo-graph form [32] for the problem (33) as follows:

$$
\begin{array}{cl}
\max _{\boldsymbol{P}, \zeta} \zeta & \\
\text { subject to } & R_{j}(\boldsymbol{P}) \geq \zeta \quad \forall j \\
& 0 \leq P_{j-1} \leq P_{j-1, \max } \quad \forall j .
\end{array}
$$

Theorem 1 The end-to-end instantaneous rate of the multi-hop FDR system is maximized (i.e., optimization problems (33) and (34) have optimal solution), if and only if the instantaneous transmission rates over all the links of the network are equal, i.e.,

$$
R_{1}(\boldsymbol{P})=R_{2}(\boldsymbol{P})=\ldots=R_{N+1}(\boldsymbol{P})=\zeta^{*}
$$

where $\zeta^{*}$ is the maximum achievable end-to-end rate.

\section{Proof See Appendix 2.}

Theorem 1 depicts the necessary condition for any solution of (33) to be optimal and any feasible solution of (34) which violates (35) only be a feasible solution which is not optimal. To find OPA vector $\boldsymbol{P}^{*}$ that achieves the maximum rate, we have to solve the system of $N+1$ nonlinear 
equations (35) with $N+2$ unknowns $\left(\boldsymbol{P}^{*}, \zeta^{*}\right)$, which is very difficult especially when $N$ is large. Thus, we turn our attention towards developing an efficient iterative algorithm to solve (34).

Sequential convex programming (SCP) is an efficient low-complex and fast-converging algorithm to solve nonconvex problems [32]. Accordingly, we devise an iterative algorithm based on SCP to determine the OPA for (34). SCP finds the local optimal solution of a difficult problem, through solving a sequence of approximate convex problems. However, it is not possible to apply SCP directly to (33), since it is not possible to find the approximate convex problem for (33). Accordingly, we use the transformed smooth optimization problem (34) for applying SCP. Notice that (33) and (34) are equivalent in the sense of optimality. The optimization problem (34) can further be transformed by writing $R_{j}(\boldsymbol{P})$ as difference of two concave functions $[33,34]$. This transformation makes it easier to determine the approximate convex problem of (34) at each iteration of the SCP [35]. Define the functions $f$ and $g$ as:

$$
\begin{aligned}
& f_{j}(\boldsymbol{P})=\log \left(\sigma_{j}^{2}+\sum_{i=0}^{N} P_{i}\left|h_{i, j}\right|^{2}\right) \\
& g_{j}(\boldsymbol{P})=\log \left(\sigma_{j}^{2}+\sum_{\substack{i=0 \\
i \neq j-1}}^{N} P_{i}\left|h_{i, j}\right|^{2}\right)
\end{aligned}
$$

Now, the functions $f$ and $g$ are concave functions of $\boldsymbol{P}$ [32] and $R_{j}(\boldsymbol{P})$ can be written as $f_{j}(\boldsymbol{P})-g_{j}(\boldsymbol{P})$. Accordingly, the optimization problem (34) can be re-casted as

$$
\begin{array}{cc}
\max _{\boldsymbol{P}, \zeta} \zeta & \\
\text { subject to } & f_{j}(\boldsymbol{P})-g_{j}(\boldsymbol{P}) \geq \zeta \quad \forall j \\
& 0 \leq P_{j-1} \leq P_{j-1, \text { max }} \quad \forall j .
\end{array}
$$

Let $\boldsymbol{P}^{n}$ be the power allocation vector at step $n$ of the iterative algorithm. The first-order Taylor series approximation $\tilde{g}_{j}\left(\boldsymbol{P}, \boldsymbol{P}^{n}\right)$ of $g_{j}(\boldsymbol{P})$ at $\boldsymbol{P}^{n}$ is given as follows:

$$
\tilde{g}_{j}\left(\boldsymbol{P}, \boldsymbol{P}^{n}\right)=g_{j}\left(\boldsymbol{P}^{n}\right)+\nabla g_{j}^{T}\left(\boldsymbol{P}^{n}\right)\left(\boldsymbol{P}-\boldsymbol{P}^{n}\right)
$$

where the gradient of $g_{j}(\boldsymbol{P})$ at $\boldsymbol{P}$ is represented as $\nabla g_{j}(\boldsymbol{P})$ and is expressed as follows:

$$
\nabla g_{j}(\boldsymbol{P})=\frac{1}{\sigma_{j}^{2}+\sum_{\substack{i=0 \\ i \neq j-1}}^{N} P_{i}\left|h_{i, j}\right|^{2}} \cdot \boldsymbol{e}_{j}
$$

Here, $\boldsymbol{e}_{j}$ is the $N+1$ dimensional column vector, $\boldsymbol{e}_{j}(j-1)=0$ and $\boldsymbol{e}_{j}(i)=\frac{\left|h_{i, j}\right|^{2}}{\ln 2}, i \neq j-1$. Further, $\tilde{g}_{j}\left(\boldsymbol{P}, \boldsymbol{P}^{n}\right)$ has the following properties with $g_{j}(\boldsymbol{P})$.

$$
\begin{aligned}
& \text { (i) } \tilde{g}_{j}\left(\boldsymbol{P}, \boldsymbol{P}^{n}\right) \geq g_{j}(\boldsymbol{P}) \forall \boldsymbol{P} \\
& \text { (ii) } \tilde{g}_{j}\left(\boldsymbol{P}^{n}, \boldsymbol{P}^{n}\right)=g_{j}\left(\boldsymbol{P}^{n}\right) \\
& \text { (iii) } \nabla \tilde{g}_{j}\left(\boldsymbol{P}^{n}, \boldsymbol{P}^{n}\right)=\nabla g_{j}\left(\boldsymbol{P}^{n}\right), \forall j
\end{aligned}
$$

Due to the slow sensitivity of the function $g_{j}(\boldsymbol{P})$ to the changes in $\boldsymbol{P}$, the approximation given by (39) is very tight over a fairly large neighborhood of $\boldsymbol{P}^{n}$. Further, property (i) arises due to the concavity of $g_{j}(\boldsymbol{P})$ and it depicts that the global information can be estimated using the local information (i.e., $g_{j}\left(\boldsymbol{P}^{n}\right)$ and $\nabla g_{j}^{T}\left(\boldsymbol{P}^{n}\right)$ ) ([32], chapter 3, $\sec$ 3.1.3).

Now, by plugging (39) into (38) and using (41), the approximated convex optimization problem can be transformed into a lower bound optimization problem to (34) as:

$$
\begin{array}{lll}
\max _{\boldsymbol{P}, \zeta} & \zeta \\
\text { s.t } \quad & f_{j}(\boldsymbol{P})-\tilde{g}_{j}\left(\boldsymbol{P}, \boldsymbol{P}^{n}\right) \geq \zeta \quad \forall j \\
& 0 \leq P_{j-1} \leq P_{j-1, \max } & \forall j .
\end{array}
$$

The constraints (42b) and (42c) form a convex set. Further, the constraint (42b) is smooth; hence, (42) is a standard convex optimization problem, which can be solved by using efficient software packages for convex optimization [36]. Algorithm 1 describes the iterative procedure to be followed for finding the OPA vector. In each iteration $n$, Algorithm 1 solves the approximate convex problem (42) and the solution obtained in iteration $n$ (i.e., $\boldsymbol{P}^{n}$ ) is used to determine the successive approximate convex problem in iteration $n+1$. The process continues until the condition on the convergence is satisfied. In the simulation, we observe that Algorithm 1 converges very quickly to the optimal solution.

$\overline{\text { Algorithm } 1 \text { Iteratve algorithm for OPA based on SCP: }}$ multi-hop FDR

\section{1: Intialization:}

- Set index $n=0$ and choose tolerance level $\epsilon>0$

- Choose $\boldsymbol{P}^{0}$, calculate $I^{0}=\min _{j}\left[f_{j}\left(\boldsymbol{P}^{0}\right)-g_{j}\left(\boldsymbol{P}^{0}\right)\right]$.
2: Repeat
3: obtain optimal solution $\hat{\boldsymbol{P}}$ by solving (42)
4: increment $n$ by 1 and assign $\boldsymbol{P}^{n}=\hat{\boldsymbol{P}}$
5: Compute $I^{n}=\min _{j}\left[f_{j}\left(\boldsymbol{P}^{n}\right)-g_{j}\left(\boldsymbol{P}^{n}\right)\right]$.
6: stop if $\left|I^{n}-I^{n-1}\right| \leq \epsilon$.

Lemma 1 The iterative Algorithm 1 described above (i) converges and (ii) the convergence point is a KKT point for the original problem (34).

Proof See Appendix 3 
As the original problem (34) is non-convex, it is not possible to prove the convergence to a global optimal point. Since the algorithm is based on SCP, which finds local optimal solutions, it may not guarantee global optimal solution to the original non-convex problem. Even if it achieves global optimality, it cannot be proved theoretically. However, from Lemma 1, the algorithm achieves a KKT point to the original problem. The computational complexity of Algorithm 1 is proportional to $O\left(\left\lceil I^{\max }-I^{0}-\frac{\epsilon}{\Delta I}\right\rceil \max \left\{(N+1)^{3}, S\right\}\right)([32]$, chapter 1 , sec 1.3.1), where $I^{\max }=\max _{\boldsymbol{P}} \min _{j} f_{j}(\boldsymbol{P})-g_{j}(\boldsymbol{P}), \Delta I=$ $\min _{n}\left[I^{n}-I^{n-1}\right]$ and $S$ is the cost of computing $f_{j}(\boldsymbol{P})$ and their first and second gradients [32]. Accordingly, the proposed iterative algorithm has affordable computational complexity and the optimization problem can be solved in polynomial time which makes it suitable for practical applications.

\subsubsection{End-to-end outage probability minimization}

Here, we formulate the OPA problem that minimizes the end-to-end outage probability of the multi-hop FDR system. Since the expressions for the end-to-end outage probability described in Sections 3.1.1 and 3.1.2 are not in a tractable form to optimize, we use the asymptotic outage probability described in Section 3.1.2. We transform the outage probability minimization into a standard geometric programming problem (GPP) and establish that the optimal solution can be obtained by solving the GPP. Consider the expression for asymptotic outage probability of multi-hop FDR system under Rayleigh fading:

$$
\hat{P}_{\text {out }, \text { asy }}^{F D R}=1-\prod_{j=1}^{N+1} \exp \left[-\frac{\Gamma_{T}}{\hat{\beta}_{j-1, j}}\left(1+\sum_{\substack{i=0 \\ i \neq j-1}}^{N} \hat{\beta}_{i, j}\right)\right]
$$

From (43), it is observed that minimization of $\hat{P}_{\text {out,asy }}^{F D R}$ is equivalent to minimization of $Q(\boldsymbol{P})=$ $\log \left(1-\hat{P}_{\text {out }, \text { asy }}^{F D R}\right)=\sum_{j=1}^{N+1} \frac{\Gamma_{T}}{\hat{\beta}_{j-1, j}}\left(1+\sum_{\substack{i=0 \\ i \neq j-1}}^{N} \hat{\beta}_{i, j}\right)=$ $\sum_{j=1}^{N+1} \frac{\Gamma_{T}}{P_{j-1} \pi_{j-1, j}}\left(\sigma_{j}^{2}+\sum_{\substack{i=0 \\ i \neq j-1}}^{N} P_{i} \pi_{i, j}\right)$. Choosing $Q(\boldsymbol{P})$ as the objective function, the OPA problem is formulated as:

$$
\min _{\boldsymbol{P}} Q(\boldsymbol{P})=\sum_{j=1}^{N+1} \frac{\Gamma_{T}}{P_{j-1} \pi_{j-1, j}}\left(\sigma_{j}^{2}+\sum_{\substack{i=0 \\ i \neq j-1}}^{N} P_{i} \pi_{i, j}\right)
$$

$$
\text { subject to } \quad 0 \leq P_{j-1} \leq P_{j-1, \max } \forall j
$$

Now, by inspecting (44), we observe the following cases: (1) the objective (44a) is a posynomial function, (2) the left hand side of the inequality constraint is a posynomial function, and (3) the right hand side of the inequality constraint is monomial [37]. Now, (44) is a GPP in standard form; GPPs can be reformulated as convex optimization problems and can be solved efficiently using standard software package for convex optimization [36].

The power allocation considered above minimizes the asymptotic outage for Rayleigh fading case. With the help of numerical and simulation results, we have verified that the OPA minimizes the outage for Nakagami fading as well, provided $m_{i, j}=m \forall i, j$. We provide the numerical results for the optimal power allocation in Section 5.

Further, the power allocation procedures described in this section can be implemented in a centralized manner. It is assumed that either I-CSI or S-CSI corresponding to various links in the multi-hop network are available at a centralized controller (i.e., either source or destination). The nodes in the network have to collect the S-CSI or I-CSI and the information should be fed back to the centralized controller. For node $F_{i}\{i \in(1, \ldots, N+1)\}$ to have the required information (i.e., either I-CSI or SCSI), all the $N+2$ nodes in the network can sequentially transmit a predefined pilot message. Under the assumption of channel reciprocity and with the broadcast nature of wireless transmissions, every other node can use the predefined pilot transmission to learn the channel. After $N+2$ pilot transmissions, every node would learn their channel to every other node. Each node communicates the acquired information to the central entity. The central entity can run Algorithm 1, compute the OPA vector, and distribute the transmit power values among the nodes in the network [38].

\subsection{Optimal power allocation for multi-hop HDR system} Here, we formulate OPA problems for multi-hop HDR system. Let $R_{j}^{H D R}(\boldsymbol{P})$ be the instantaneous transmission rate corresponding to the link $j, j=1,2, \ldots N+1$. From (27), the achievable end-to-end instantaneous transmission rate of the multi-hop HDR system is computed using the formula given as follows:

$$
\begin{array}{r}
R^{H D R}(\boldsymbol{P})=\min _{j}\left(R_{j}^{H D R}(\boldsymbol{P})\right), \quad j=1,2, \ldots, N+1 \\
=\min _{j} \frac{1}{2} \log \left(1+\frac{P_{j-1}\left|h_{j-1, j}\right|^{2}}{\sigma_{j}^{2}+\sum_{\substack{i=0 \\
p \neq j-1}}^{\left\lfloor\frac{N-q}{2}\right\rfloor} P_{p}\left|h_{p, j}\right|^{2}}\right)
\end{array}
$$

The OPA problem for the multi-hop HDR that maximizes the end-to-end rate is formulated as

$$
\begin{aligned}
& \max _{\boldsymbol{P}} \min _{j} R_{j}^{H D R}(\boldsymbol{P}) \\
& \text { subject to } \quad 0 \leq P_{j-1} \leq P_{j-1, \text { max }} \forall j .
\end{aligned}
$$


The optimization problem (46) is similar to the problem (33). Optimal solution for problem (46), i.e., OPA for multi-hop HDR system, can be determined by following the procedure adopted for the multi-hop FDR system.

Consider the asymptotic outage probability for multihop HDR system $\bar{P}_{\text {out,asy }}^{H D R}$. Now, minimization of $\bar{P}_{\text {out,asy }}^{H D R}$ given by (32) is equivalent to $Q^{H D R}(\boldsymbol{P})=-\log \left(1-\bar{P}_{\text {out }, \text { asy }}^{H D R}\right)$. Choosing $Q^{H D R}(\boldsymbol{P})$ as the objective function for outage probability minimization, the OPA problem for multi-hop HDR system is formulated as:

$$
\min _{\boldsymbol{P}} Q^{H D R}(\boldsymbol{P})=\sum_{j=1}^{N+1} \frac{\gamma_{T}}{P_{j-1} \pi_{j-1, j}}\left(\sigma_{j}^{2}+\sum_{\substack{i=0 \\ p \neq j-1}}^{\left\lfloor\frac{N-q}{2}\right\rfloor} P_{p} \pi_{p, j}\right)
$$

subject to $0 \leq P_{j-1} \leq P_{j-1, \max } \forall j$

The optimization problem (47) is similar to the problem (44). Optimal solution for problem (47), i.e., OPA for multi-hop HDR system, can be determined by following the procedure adopted for the multi-hop FDR system.

\section{Results and discussion}

This section describes the results for the outage probability and related metrics of multi-hop FDR and HDR systems under the proposed power allocation algorithm. We assume a linear network, where the distance between the adjacent nodes are set as equal. Further, the normalized distance between the nodes $F_{0}$ and $F_{N+1}$ (normalized with respect to a reference distance $d_{0}$ ) is set equal to 10 and it is assumed to be the same regardless of the number of relay nodes, $N$ in the network. We set $\pi_{i, j}=G d_{i j}^{-\eta}$, where $G$ is the propagation constant, $\eta$ is the path loss exponent, and $d_{i j}$ is the distance between relay nodes $F_{i}$ and $F_{j}$. We select $\eta$ as 3 and the target rate $r=0.1 \mathrm{bps} / \mathrm{Hz}$. The maximum available transmit power and the average power due to RSI are the same for all the nodes, i.e., $P_{j, \max }=P_{\max }$ and $\pi_{j, j}=\pi_{0} \forall j$. Furthermore, we assume the Nakagami parameter $m_{i, j}=m$ and the noise power to be unity. Under UPA, it is assumed that all the nodes in the network operate at $P_{\max }$. Extensive Monte Carlo simulations have been performed to corroborate the analytical results. In the implementation of the proposed Algorithm 1, the tolerance level $\epsilon$ has been chosen as equal to $10^{-6}$.

In Fig. 2, the analytical and simulation results for outage probability of four-hop FDR/HDR system has been drawn against $P_{\max }$ assuming UPA (i.e., equal transmit power for all the nodes). As $P_{\max }$ increases, the outage probability of both FDR and HDR systems decreases. However, for higher values of $P_{\max }$, the outage probability exhibits a floor behavior, due to the higher level of IRI. Figure 2 also shows the impact of RSI $\left(\pi_{0}\right)$ on the outage probability of FDR system. When the RSI is comparatively lower, FDR performs significantly better than HDR due to the fact that HDR systems need to achieve double the target rate of FDR, i.e., for a given rate $r$, the threshold SINR required for HDR system is higher than that of FDR [4]. However, when RSI is higher, the range of $P_{\max }$ values over which FDR performs superior than HDR decreases. For low and medium values of $P_{\max }$, FDR continues to exhibit improved performance than HDR. However, when $P_{\text {max }}$ is

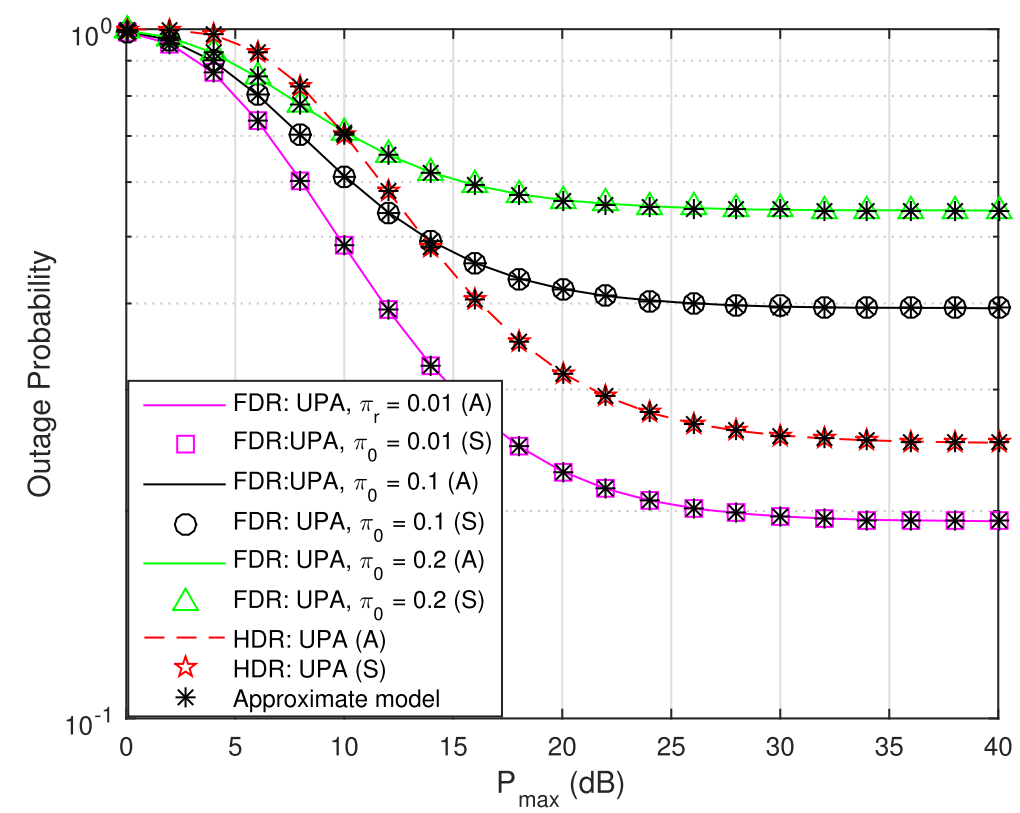

Fig. 2 Four-hop FDR/HDR systems: outage probability vs $P_{\max }$, under UPA ( $m=1$, A analytical, $S$ simulation) 
higher, FDR becomes inferior to HDR because of the RSI and higher amount of IRI in the FDR system as compared to the spectral efficient HDR system [23] considered in this paper. Figure 2 also shows the results evaluated with approximate outage probability expressions given by (22) and (30) for the multi-hop FDR and HDR systems respectively. It can be observed that the approximate outage probability model is highly accurate, i.e., the outage probability corresponding to the approximate model is within $5 \%$ of the exact outage probability values.

In Fig. 3, we plot the analytical and simulation results for the outage probability of four-hop FDR network under UPA and the proposed OPA for different values of $m$, the Nakagami fading parameter, while Fig. 4 shows the corresponding results for HDR systems. The results show that the outage probability of FDR and HDR systems improve significantly under OPA compared to UPA, especially for higher values of $P_{\max }$. When $P_{\max }$ is very high, FDR/HDR systems with conventional UPA suffer from very high IRI whereas the proposed OPA strategy allocates the transmit power among the nodes in such a way that the effect of IRI is reduced, thereby improving the outage performance. With $P_{\max }=30 \mathrm{~dB}$ and $m=1$, the optimal power allocation vector (in $\mathrm{dB}$ ) has been obtained as $\boldsymbol{P}^{*}=[30,28.88,25.46,23.05]$; it provides approximately $30 \%$ reduction in outage probability for the FDR system as compared to that achievable under UPA. Similarly, with $P_{\max }=30 \mathrm{~dB}$ and $m=1$, the corresponding optimal power allocation (in $\mathrm{dB}$ ) for the HDR system is given by $\boldsymbol{P}^{*}=[30,30,23.45,23.45]$; it provides approximately $49 \%$ reduction in outage probability for the HDR system as compared to that achievable under UPA. Further, as $m$ increases, the percentage reduction in outage probability has been observed to be higher, i.e., when $m=2$, the outage probability reduces by $69 \%$ (89\%) for FDR (HDR) system under OPA, as compared to the corresponding system under UPA.

In Fig. 5, we plot the outage probability of the FDR system against the number of relays $(N)$ in the network. Since the distance between source and destination is assumed to be constant, the mean signal power over each link depends on $N$. As shown, OPA always has an edge over UPA when outage performance is considered. Initially, the outage probability decreases as $N$ is increased owing to the reduction in hop length. However, when $N$ increases beyond a certain value, the outage probability significantly increases. This happens due to higher amount of IRI in the system as $N$ becomes larger. From the graphs, it is also clear that an optimum value for $N$ exists that leads to minimum outage probability and the corresponding value of $N$ depends on $P_{\max }$.

In the next set of simulations, we consider perfect knowledge of I-CSI. The channel gain matrix $\boldsymbol{H}=\left[\left|h_{i, j}\right|^{2}\right]$ has been assumed as follows:

$\left[\begin{array}{llll}0.0741 & 0.0026 & 0.0001 & 0.0020 \\ 0.0239 & 0.1349 & 0.0056 & 0.0003 \\ 0.0830 & 0.0197 & 0.2925 & 0.0052 \\ 0.0004 & 0.0607 & 0.0036 & 0.0283\end{array}\right]$

where each element $\left|h_{i, j}\right|^{2}$ is generated randomly as Gamma $\left(m_{i, j}, \frac{\pi_{i, j}}{m_{i, j}}\right)$. The power allocation vector that

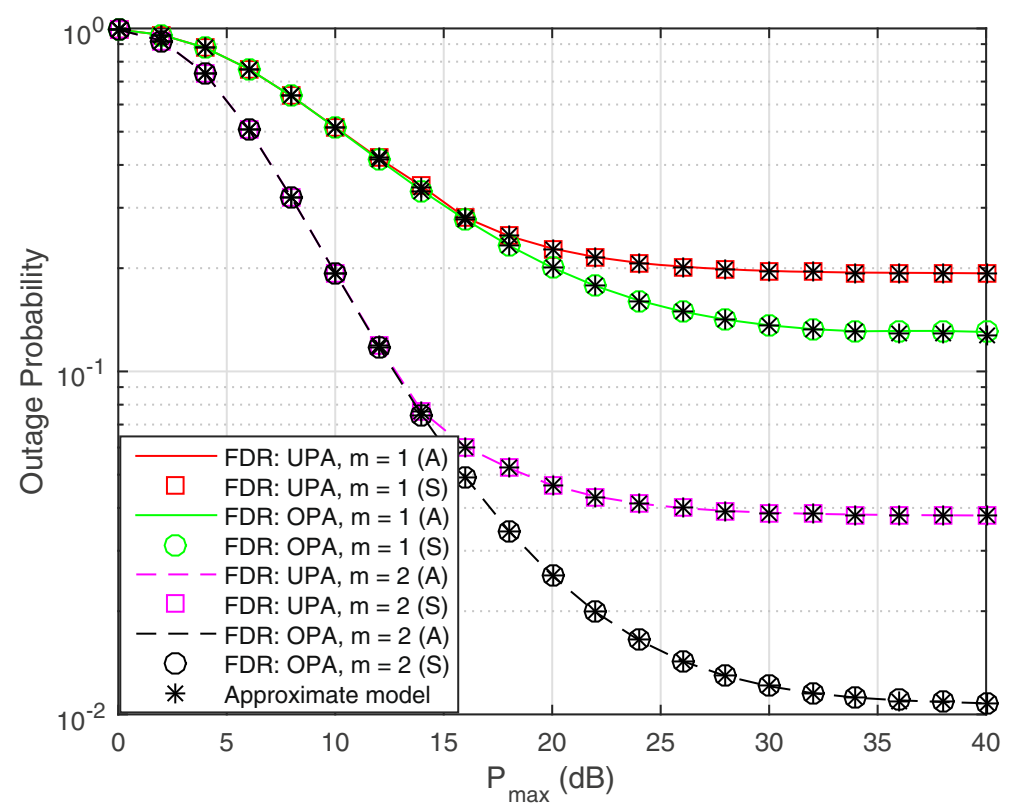

Fig. 3 Outage probability performance of four-hop FDR system vs $P_{\max }$ under UPA and OPA, for different values of $m: \pi_{0}=0.01$ 


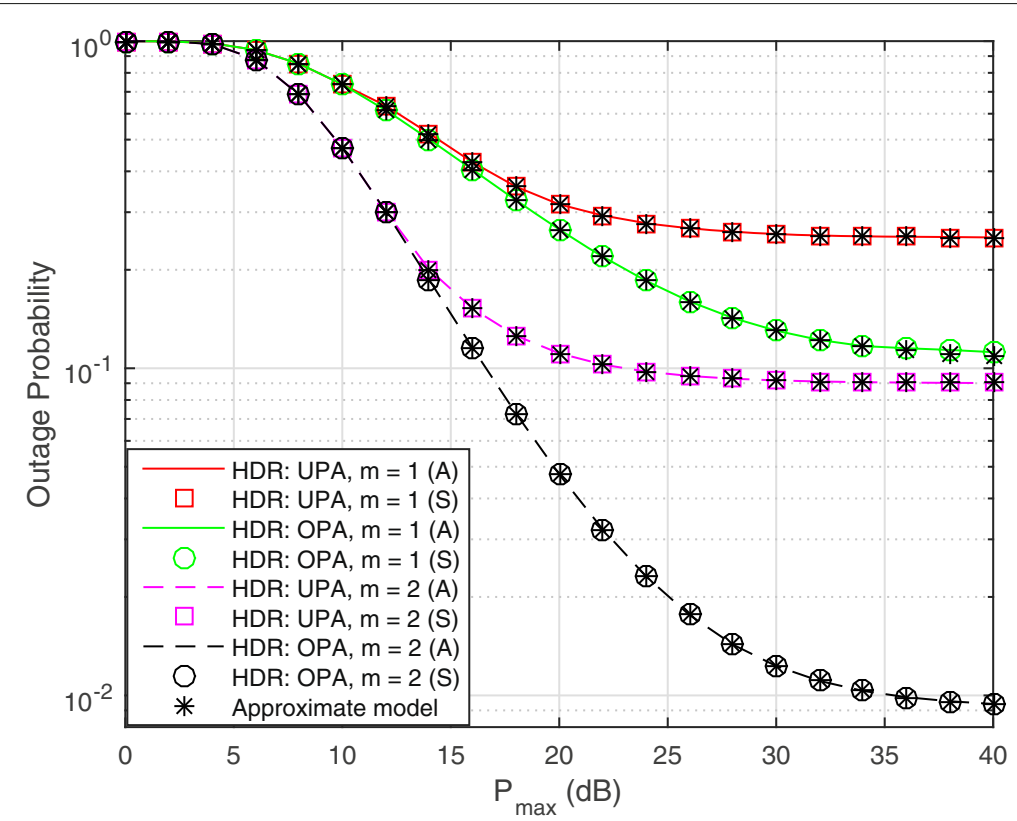

Fig. 4 Outage probability of four-hop HDR system vs $P_{\max }$ under UPA and OPA, for different values of $m: N=3$

maximizes the end-to-end instantaneous rate has been determined by using Algorithm 1.

Figure 6 illustrates the convergence of Algorithm 1 for four-hop FDR and HDR systems under I-CSI. It can be seen that the proposed algorithm converges very fast and the optimal solution can be obtained with a few number of iterations. For example, when $\boldsymbol{P}^{0}=0.5 P_{\max }$, the algorithm converges within three iterations for the FDR system and the maximum end-to-end instantaneous rate $R(\boldsymbol{P})$ obtained is equal to $2.1999 \mathrm{bps} / \mathrm{Hz}$ and optimal power (in $\mathrm{dB}$ ) allocation vector is obtained as $\boldsymbol{P}^{*}=$ $[40,38.06,27.86,35.20]$. Further, for this $\boldsymbol{P}^{*}$, the maximum end-to-end instantaneous rate has been observed to be equal for all the individual links, i.e., $R_{j}(\boldsymbol{P})=2.1999$ bps $/ \mathrm{Hz}$. This corroborates the necessary condition for optimality (35) described in Section 4. Similarly, in the case of HDR systems, the maximum end-to-end instantaneous rate $R^{H D R}(\boldsymbol{P})$ is obtained as $1.8764 \mathrm{bps} / \mathrm{Hz}$ which

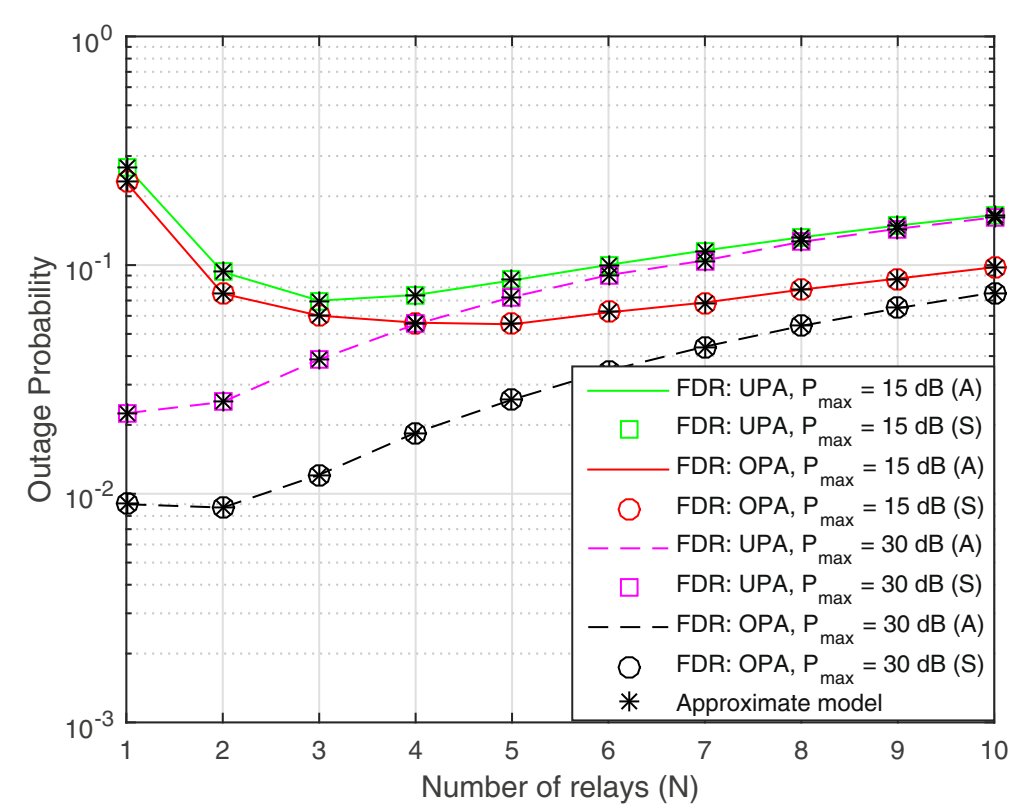

Fig. 5 Outage probability performance of multi-hop FDR system vs $N$ : $\pi_{0}=0.01$ and $m=2$ 


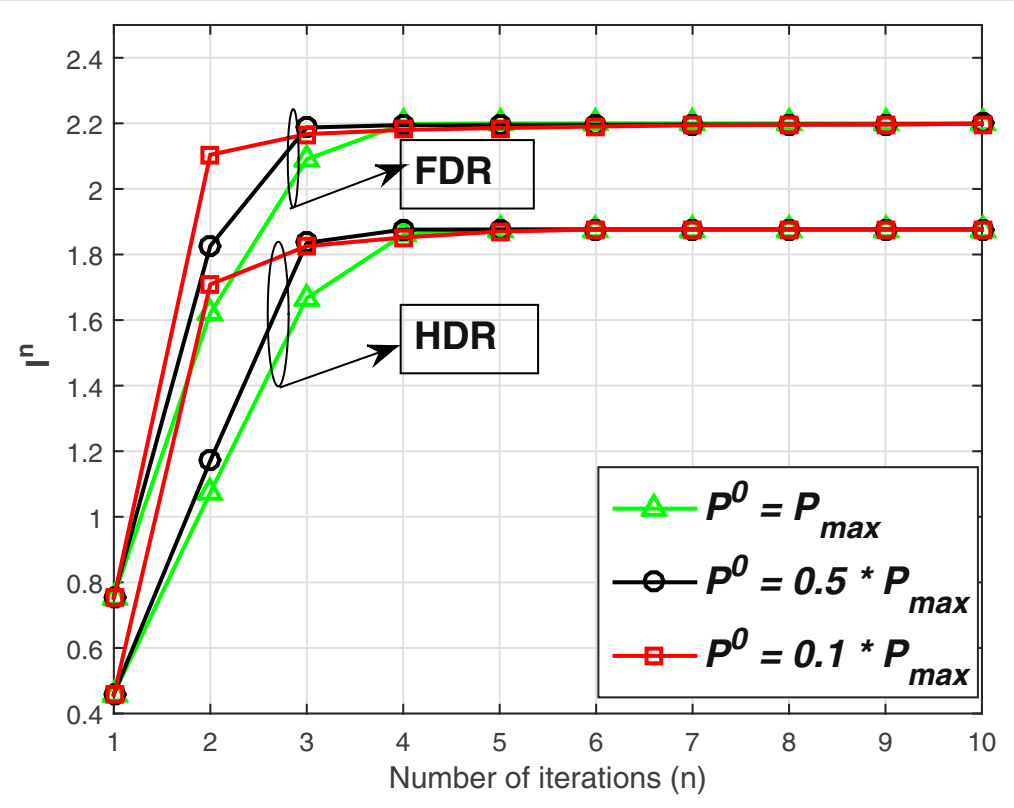

Fig. 6 Convergence of Algorithm 1 for FDR/HDR systems under I-CSI: $P_{\max }=40 \mathrm{~dB}, m=1, \pi_{0}=0.01$, and $\mathrm{N}=3$

corresponds to the optimal power (in $\mathrm{dB}$ ) allocation vector $\boldsymbol{P}^{*}=[38.91,40,25.14,32.46]$. Further, the maximum instantaneous rate over each link has been observed to be equal, i.e., $R_{j}^{H D R}(\boldsymbol{P})=1.8764 \mathrm{bps} / \mathrm{Hz}(j=1,2,3,4)$ which validates (35).

Figure 7 depicts the comparison of the maximum endto-end instantaneous rate that can be achieved for fourhop FDR and HDR systems as a function of $P_{\text {max }}$ under the proposed OPA strategy. The end-to-end instantaneous rate that can be obtained under UPA is also shown. The results confirm that the proposed OPA improves the endto-end instantaneous transmission rate for both FDR and HDR systems, especially for higher values of $P_{\max }$. When $P_{\max }=30 \mathrm{~dB}$, the percentage improvement in end-to-end rate is $174 \%$ for the FDR system and $224 \%$ for the HDR system as compared to the corresponding systems with UPA. Notice that, initially, the end-to-end instantaneous rate increases as $P_{\max }$ is increased; however, it remains

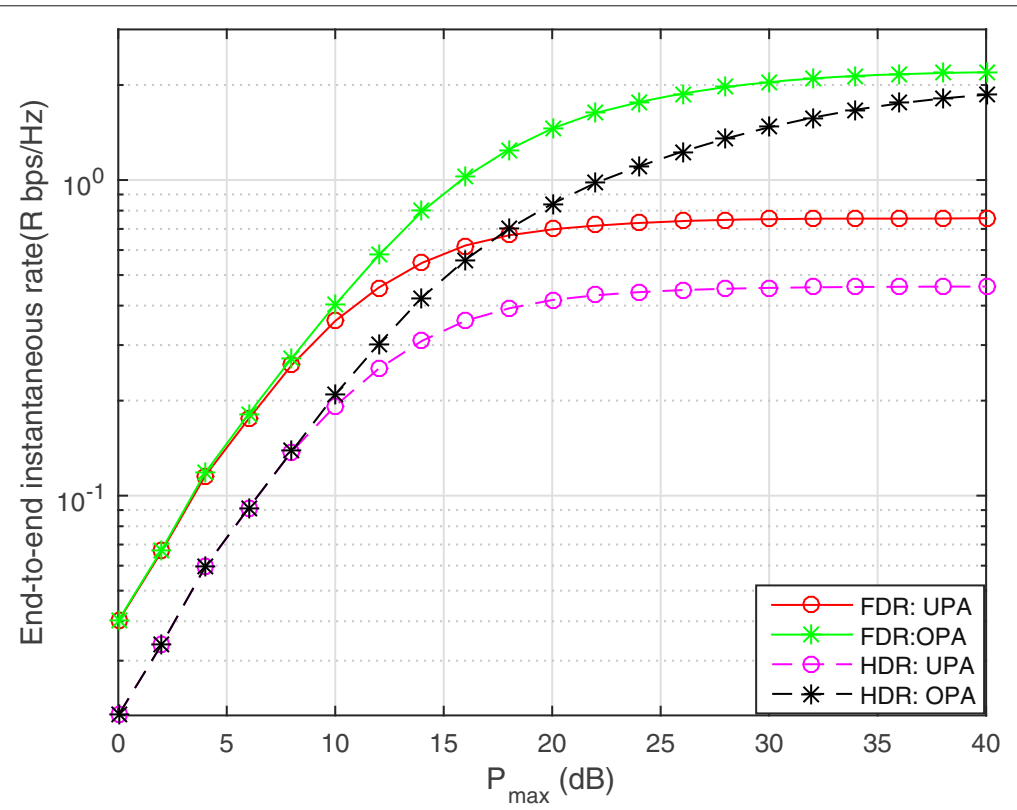

Fig. 7 End-to-end instantaneous rate comparison under UPA and OPA for FDR/HDR systems: $m=1, \pi_{0}=0.01$, and $N=3$ 
constant for higher values of $P_{\max }$ for both FDR as well as HDR systems, owing to the higher levels of IRI arising out of the increased $P_{\max }$.

Figure 8 compares the speed of convergence of SCP and bisection-based algorithms (i.e., Algorithms 1 and 2 given in Appendix 4) respectively considered for the rate maximization problem in this paper. Notice that both algorithms converge to the same optimal point for HDR as well as FDR systems. However, it is observed that SCP-based algorithm converges faster than bisection-based method for a given tolerance level $\epsilon$. Specifically, it can be observed that the SCP-based algorithm converges within four iterations while the bisection-based algorithm converges to the optimal point only after 22 iterations. This implies that the SCP-based OPA algorithm has lower computational complexity as compared to the bisection-based OPA algorithm.

Since conventional HDR networks use multi-phase relaying, the end-to-end delay experienced is larger, which considerably affects the spectral efficiency. In addition to the delay introduced by multi-phase transmission, each relay node introduces processing delay as well. However, in multi-hop FDR networks, since the relay nodes perform simultaneous transmission and reception, the processing delay at each node and the end-to-end delay would be much lower than that of HDR networks. The higher end-to-end delay will have significant influence on the spectral efficiency of multi-hop HDR networks, while its impact would be insignificant in the case of FDR networks.

\section{Conclusions}

In this paper, we have derived closed-form expression for the end-to-end outage probabilities of multi-hop FDR and HDR networks in Nakagami- $m$ fading channel. We have also derived approximate expressions for the outage probability which were observed to be highly accurate. We then considered optimal power allocation (OPA) to maximize end-to-end rate with constraints on maximum transmit power at each node. It was shown that the OPA problem is non-convex. Then, based on sequential convex programming, we have proposed an iterative algorithm to solve the optimization problem. With the help of extensive simulations, it was established that the algorithm converges very fast and optimal solution (i.e., OPA vector) can be obtained with a few number of iterations. We also considered OPA to minimize the end-to-end outage probability with constraints on maximum transmit power at each node. We employed geometric programming to solve the OPA problem. Further, it was shown that the proposed OPA strategies would lead to significant improvements in end-to-end outage probability and data rate performance of multi-hop FDR/HDR systems as compared to uniform power allocation strategy.

\section{Methods/Experimental}

The purpose of this study is to investigate and optimize the outage and rate performance of the multi-hop FDR system. The system consists of a source node, destination node, and $N$ relay nodes. The relay nodes operate in full duplex mode. The channels between the nodes are

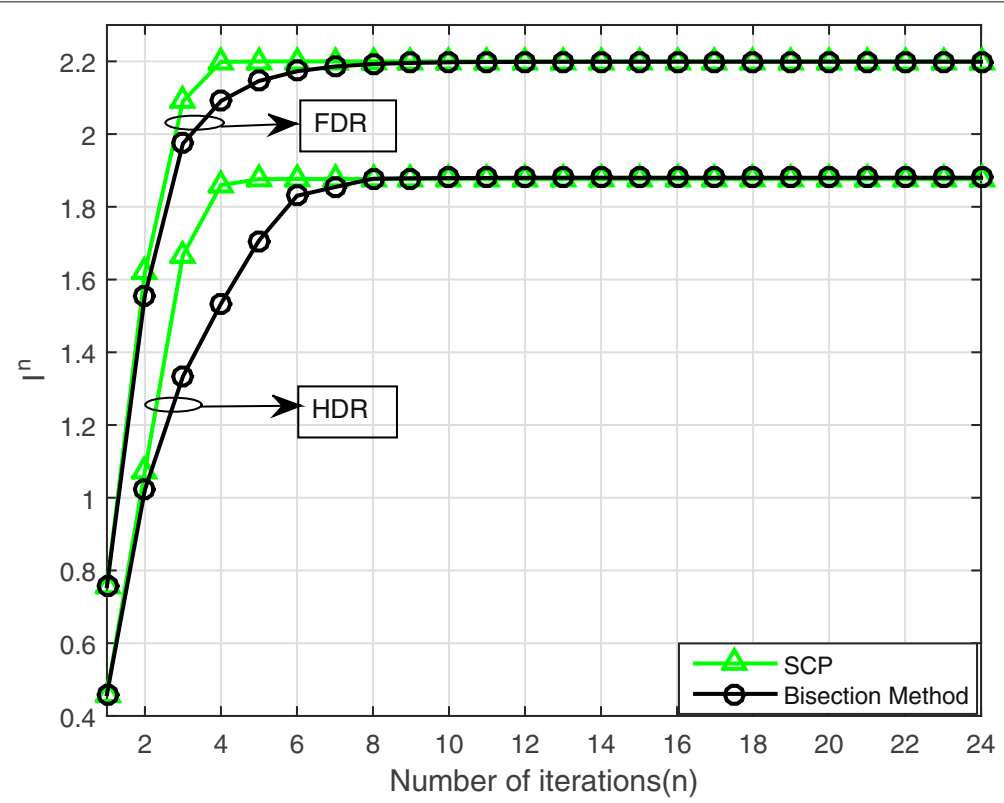

Fig. 8 Convergence of Algorithm 1 and Algorithm 2 for FDR/HDR systems with rate maximization: $P_{\max }=30 \mathrm{~dB}, m=1, \pi_{0}=0.01, P^{0}=P_{\text {max }}$, $I=0, u=3$, and $N=3$ 
assumed to follow Nakagami- $m$ fading, and it is assumed that the RSI channel has Nakagami- $m$ fading characteristics. Further, closed-form expressions for end-to-end outage probability are provided, power optimization is based on SCP and GPP is performed. The investigations are repeated for spectral efficient HDR system. Since the relay node in HDR system needs two orthogonal channels for transmission and reception, for fair comparison, the target rate of HDR system is chosen as twice that of FDR system.

\section{Appendix 1: Derivation of (18)}

Assume that the double integral term in (17) is represented as $\theta$, which can be written as follows:

$$
\theta=\int_{0}^{\infty} y^{M-1} e^{-a y} \int_{y+1}^{\infty} w^{N-1} e^{-b w} d w d y
$$

Using incomplete Gamma function [40], we get

$$
\theta=\frac{1}{b^{N}} \int_{0}^{\infty} y^{M-1} e^{-a y} \times \Gamma(N, b(y+1)) d y
$$

With the help of series expansion for the incomplete Gamma function and by using binomial expansion of $(1+y)^{n}$, we get

$\theta=(N-1) ! e^{-b} \sum_{n=0}^{N-1} \frac{b^{n-N}}{n !} \int_{0}^{\infty} y^{M-1} e^{-(a+b) y} \sum_{r=0}^{n}\left(\begin{array}{l}n \\ r\end{array}\right) y^{r} d y$

Now, $\theta$ given by (50) can be written as follows by using the definition of Gamma function:

$\theta=\Gamma(N) e^{-b} \sum_{n=0}^{N} \frac{b^{n-N}}{\Gamma(n+1)} \sum_{r=0}^{n}\left(\begin{array}{l}n \\ r\end{array}\right)(a+b)^{-(M+r)} \Gamma(M+r)$

Notice that here $M=m_{i, j}, a=\frac{1}{\beta_{i, j}}, N=m_{j-1, j}$ and $b=\frac{\Gamma_{T}}{\beta_{j-1, j}}$. By substituting the resulting expression for $\theta$ in (17), and after mathematical simplification, we get the following:

$$
\begin{aligned}
\operatorname{Pr}\left(\Gamma_{j}(\boldsymbol{P}) \geq \Gamma_{T}\right)= & \sum_{\substack{i=0 \\
i \neq j-1}}^{N} \sum_{k=1}^{m_{i, j}} \sum_{n=0}^{m_{j-1, j}-1} \sum_{r=0}^{n} \frac{\Xi(i, k)}{n !} e^{-\frac{\Gamma_{T}}{\beta_{j-1, j}}} \\
& \times\left(\begin{array}{c}
n \\
r
\end{array}\right) \beta_{i, j}^{r}\left(\frac{\Gamma_{T}}{\beta_{j-1, j}}\right)^{n}\left(1+\frac{\Gamma_{T} \beta_{i, j}}{\beta_{j-1, j}}\right)^{-\left(m_{i, j}+r\right)} \frac{\Gamma\left(m_{i, j}+r\right)}{\Gamma\left(m_{i, j}\right)}
\end{aligned}
$$

Finally, substituting (52) in (9), we get (18).

\section{Appendix 2: Proof of Theorem 1}

Let $\zeta^{*}$ be the maximum achievable end-to-end rate. By contradiction, assume that $R_{k}(\boldsymbol{P})>\zeta^{*}$ for some $k$. Now, strictly decrease $P_{k-1}$ by $\delta_{k-1}$ such that $R_{k}(P)>\zeta^{*}$. Decrementing $P_{k-1}$ results in reduction of interference at
$F_{j}, j \neq k$. Therefore, there exists a positive $\epsilon_{j}$ such that $R_{j}(\boldsymbol{P}) \geq \zeta^{*}+\epsilon_{j}>\zeta^{*}, \forall j$. This contradicts with the assumption that $\zeta^{*}$ is optimal. Hence, at the optimal point, instantaneous rate of all the links of the multi-hop FDR network are equal.

\section{Appendix 3: Proof of Lemma 1}

(i) The constraint set of (34) is compact and hence closed and bounded. From Algorithm 1, we have the sequence $\left\{I^{n}\right\}$ where

$$
\begin{aligned}
I^{n} & =\min _{j}\left[f_{j}\left(\boldsymbol{P}^{n}\right)-g_{j}\left(\boldsymbol{P}^{n}\right)\right] \\
& \leq \min _{j}\left[f_{j}\left(\boldsymbol{P}^{n+1}\right)-\left(g_{j}\left(\boldsymbol{P}^{n}\right)+\nabla g_{j}^{T}\left(\boldsymbol{P}^{n}\right)\left(\boldsymbol{P}^{n+1}-\boldsymbol{P}^{n}\right)\right)\right] \\
& \leq \min _{j}\left[f_{j}\left(\boldsymbol{P}^{n+1}\right)-g_{j}\left(\boldsymbol{P}^{n+1}\right)\right]=I^{n+1}
\end{aligned}
$$

In (53), the first inequality arises due to the fact that $P^{n+1}$ is an optimal solution of (42) in $n+1$ th step, thus the obtained value of $f_{j}\left(\boldsymbol{P}^{n+1}\right) \geq f_{j}\left(\boldsymbol{P}^{n}\right)$. Further, the second inequality follows from the concavity of $g_{j}(\boldsymbol{P})$.

Now, the sequence $I^{n}$ is monotonic increasing sequence on a compact set and hence bounded. By Cauchy theorem, the sequence $\left\{I^{n}\right\}$ converges.

(ii) The convergence of Algorithm 1 implies $I^{n}=I^{n+1}$ in the limit. From (i) Algorithm 1 converges, then the convergence point is a KKT solution. Hence, $\boldsymbol{P}^{n+1}$ is a KKT point of (42).

The Lagrangian dual for (42) can be written as

$$
\begin{aligned}
L_{1}= & \zeta+\sum_{j=1}^{N+1} \lambda_{j}\left(f_{j}(\boldsymbol{P})-\tilde{g}_{j}\left(\boldsymbol{P}, \boldsymbol{P}^{n}\right)-\zeta\right) \\
& +\sum_{j=1}^{N+1} \alpha_{j}\left(P_{j-1, \max }-P_{j-1}\right)
\end{aligned}
$$

From (i) above, at the optimal point $\boldsymbol{P}^{n+1}=\boldsymbol{P}^{n}$. Now, the KKT conditions at the optimal point $\left(\boldsymbol{P}^{n+1}, \zeta^{n+1}\right)$ and using (41) implies

$$
\begin{aligned}
\frac{\partial L_{1}\left(\boldsymbol{P}^{n+1}, \zeta^{n+1}\right)}{\partial P_{j-1}}= & \sum_{j=1}^{N+1} \lambda_{j} \frac{\partial\left(f_{j}\left(\boldsymbol{P}^{n+1}\right)-g_{j}\left(\boldsymbol{P}^{n+1}\right)\right)}{\partial P_{j-1}} \\
& +\sum_{j=1}^{N+1} \alpha_{j} \frac{\partial\left(P_{j-1, \max }-P_{j-1}\right)}{\partial P_{j-1}}=0 \forall j
\end{aligned}
$$

$$
\begin{aligned}
& \frac{\partial L\left(\boldsymbol{P}^{n+1}, \zeta^{n+1}\right)}{\partial \zeta}=1-\sum_{j=1}^{N+1} \lambda_{j}=0 \\
& \left(\left(f_{j}\left(\boldsymbol{P}^{n+1}\right)-g_{j}\left(\boldsymbol{P}^{n+1}\right)\right)-\zeta\right) \lambda_{j}=0 \forall j \\
& \left(P_{j-1, \max }-P_{j-1}\right) \alpha_{j}=0 \forall j
\end{aligned}
$$


Now, the conditions (55)-(58) are same as the KKT optimality conditions for the problem (38). Thus, $\boldsymbol{P}^{n+1}$ is the KKT point of the optimization problem (34). We complete the proof.

\section{Appendix 4: Power allocation based on bisection method}

In this section, we investigate an iterative algorithm for OPA based on bisection method. Though the optimization problem (34) is non-convex in the original form, it can be transformed to a quasi-concave optimization problem [32, 39]. Since, $\log ($.$) is a monotonic increasing$ function, (34) is equivalent to the following optimization problem:

$$
\begin{gathered}
\max _{\boldsymbol{P}} \min _{j}\left\{\Gamma_{j}(\boldsymbol{P})=\frac{P_{j-1}\left|h_{j-1, j}\right|^{2}}{\sigma_{j}^{2}+\sum_{\substack{i=0 \\
i \neq j-1}}^{N} P_{i}\left|h_{i, j}\right|^{2}}\right\} \\
\text { subject to } \quad 0 \leq P_{j-1} \leq P_{j-1, \max } \forall j .
\end{gathered}
$$

This is known as generalized fractional programming, where $\Gamma_{j}(\boldsymbol{P})$ is a linear fractional function and hence it is a quasi-concave function ([32], chapter 3, sec 3.4.1); further minimum of quasi-concave functions is also a quasiconcave function [[32], chapter 3, sec 3.4.4]. Accordingly, (59) is a quasi-concave optimization problem and it can further be transformed by introducing an auxiliary variable $\tau$, as follows:

Algorithm 2 Iteratve algorithm for OPA based on Bisection method: multi-hop FDR

\section{1: Intialization:}

- Given an interval $[l, u], \epsilon_{0}=u-l$ and tolerance level $\epsilon>0$

\section{2: Repeat}

3: $\tau=(u+l) / 2$

4: given $\tau$, solve the concave feasibility problem

$$
\begin{aligned}
& \text { find } \boldsymbol{P} \\
& \text { subject to } P_{j-1}\left|h_{j-1, j}\right|^{2} \geq \tau\left(\sigma_{j}^{2}+\sum_{\substack{i=0 \\
i \neq j-1}}^{N} P_{i}\left|h_{i, j}\right|^{2}\right) \\
& 0 \leq P_{j-1} \leq P_{j-1, \max } \forall j, \quad \tau>0
\end{aligned}
$$

5: if feasible $l=\tau$; if infeasible $u=\tau$

6: stop if $|u-l| \leq \epsilon$.

$$
\begin{gathered}
\max _{\boldsymbol{P}, \tau} \tau \\
\text { subject to } P_{j-1}\left|h_{j-1, j}\right|^{2} \geq \tau\left(\sigma_{j}^{2}+\sum_{\substack{i=0 \\
i \neq j-1}}^{N} P_{i}\left|h_{i, j}\right|^{2}\right) \\
\quad 0 \leq P_{j-1} \leq P_{j-1, \text { max }} \forall j, \quad \tau>0
\end{gathered}
$$

Now, (60) is a quasi-concave optimization problem, whose solution can be determined by solving sequence of concave feasibility problems with the help of bisection search on $\tau$. Algorithm 2 presents the bisection method to determine the OPA vector.

Algorithm 2 converges in $\log \left(\epsilon_{0} / \epsilon\right)$ iterations, each iteration involved in solving a concave feasibility problem (60) for a given value of $\tau$. Accordingly, the algorithm has the computational complexity proportional to $O\left(\log \left(\epsilon_{0} / \epsilon\right) \max \left\{(N+1)^{3}\right\}\right)$ ([32], chapter 4 , Section 4.2).

\begin{abstract}
Abbreviations
AF: Amplify-and-forward; DF: Decode-and-forward; FD: Full duplex; FDR: Full duplex relaying; HD: Half duplex; HDR: Half duplex relaying; I-CSI: Instantaneous channel state information; IRI: Inter-relay interference; KKT: Karush-KuhnTucker; OPA: Optimal power allocation; RSI: Residual self-interference;

S-CSI: Statistical channel state information; UPA: Uniform power allocation
\end{abstract}

\section{Authors' contributions}

It is a combined work of two authors. Both authors read and approved the final manuscript.

\section{Competing interests}

The authors declare that they have no competing interests.

\section{Publisher's Note}

Springer Nature remains neutral with regard to jurisdictional claims in published maps and institutional affiliations.

Received: 14 December 2017 Accepted: 19 July 2018

Published online: 09 August 2018

\section{References}

1. MO Hasna, M-S Alouini, Outage probability of multihop transmission over Nakagami fading channels. IEEE Commun. Lett. 7(5), 216-218 (2003)

2. GK Karagiannidis, TA Tsiftsis, RK Mallik, Bounds for multihop relayed communications in Nakagami- $m$ fading. IEEE Trans. Commun. 54(1), 18-22 (2006)

3. G Liu, et al, In-band full-duplex relaying: a survey, research issues and challenges. IEEE Commun. Surv. Tutor. 17(2), 500-524 (2015)

4. TKwon, et al, Optimal duplex mode for DF relay in terms of outage probability. IEEE Trans. Veh. Technol. 59(7), 18-22 (2010)

5. M Duarte, C Dick, A Sabharwal, Experiment-driven characterization of full-duplex wireless systems. IEEE Trans. Wirel. Commun. 11(12), 4296-4307 (2012)

6. E Everett, D Dash, C Dick, A Sabharwal, in Proc. 49th AACCCC. Self-interference cancellation in multi-hop full-duplex networks via structured signaling (IEEE, Monticello, 2011), pp. 1619-1626

7. K Dhaka, RK Mallik, R Schober, in Proc. ICC. Optimal power allocation in a multi-hop decode-and-forward communication system (IEEE, Ottawa, 2012), pp. 4458-4462

8. SS Ikki, S Aissa, Multihop wireless relaying systems in the presence of cochannel interferences: performance analysis and design optimization. IEEE Trans. Veh. Technol. 61(2), 566-573 (2012) 
9. TP Do, I Song, YH Kim, Optimization of power allocation and relay position in multihop relay networks over generalized fading channels. IEEE Trans. Veh. Technol. 64(11), 5031-5041 (2015)

10. H Shen, W Xu, C Zhao, QoS constrained optimization for multi-antenna AF relaying with multiple eavesdroppers. IEEE Signal Process. Lett. 22(12), 2224-2228 (2015)

11. H Shen, W Xu, C Zhao, A semi-closed form solution to MIMO relaying optimization with source-destination link. IEEE Signal Process. Lett. 23(2), 247-251 (2016)

12. TKr Baranwal, DS Michalopoulos, R Schober, Outage analysis of multihop fullduplex relaying. IEEE Commun. Lett. 17(1), 63-66 (2013)

13. PK Sharma, P Garg, Performance analysis of full duplex decode-and-forward cooperative relaying over Nakagami- $m$ fading channels, Trans. Emerg. Telecommun. Technol. 25(9), 905-913 (2014)

14. S Han, L Chen, W Meng, C Li, in Proc. ICCC. Outage probability of multi-hop full-duplex DF relay system over Nakagami- $m$ fading channels (IEEE, Shenzhen, 2015), pp. 1-6

15. R Katla, AV Babu, in Proc. INDICON. Outage performance of multihop full duplex relaying system over Nakagami-m fading channels (IEEE, Bangalore, 2016), pp. 1-6

16. TRiihonen, S Werner, R Wichman, Hybrid full-duplex/half-duplex relaying with transmit power adaptation. IEEE Trans. Wireless Commun. 10(9), 3074-3085 (2011)

17. LJ Rodriguez, NH Tran, T Le-Ngoc, Optimal power allocation and capacity of full-duplex AF relaying under residual self-interference. IEEE Wireless Commun. Lett. 3(2), 233-236 (2014)

18. $B$ Y u, L Yang, X Cheng, R Cao, Power and location optimization for full-duplex decode-and-forward relaying. IEEE Trans. Commun. 63(12), 4743-4753 (2015)

19. S Li, Y Kun, M Zhou, J Wu, L Song, Y Li, H Li, Full-duplex amplify-and-forward relaying: power and location Optimization. IEEE Trans. Veh. Technol. 60(9), 8458-8468 (2017)

20. S Li, et al, in Proc. 19th WPMC. Joint power allocation and location optimization for full-duplex amplify-and-forward relay networks (IEEE, Shenzhen, 2016), pp. 250-255

21. D Rajan, Optimum number of hops in linear multihop wireless networks. Int. J. Adv. Eng. Sci. Appl. Math. 5(1), 32-42 (2013)

22. B Mahboobi, M Ardebilipour, Joint power allocation and routing in full-duplex relay network: an outage probability approach. IEEE Commun. Lett. 17(8), 1497-1500 (2013)

23. G Farhadi, JM Cioffi, Spectral efficient multihop relaying based on alternate transmission. IEEE Trans. Wireless Commun. 10(11), 3601-3606 (2011)

24. JN Laneman, DNC Tse, GW Wornell, Cooperative diversity in wireless networks: efficient protocols and outage behavior. IEEE Trans. Inf. Theory. 50, 3062-3080 (2004)

25. R Pabst, et al, Relay-based deployment concepts for wireless and mobile broadband radio. IEEE Commun. Magazine. 42(9), 80-89 (2004)

26. Y Yang, H Hu, J Xu, G Mao, Relay technologies for WiMax and LTE-advanced mobile systems. IEEE Commun. Magazine. 47(10), 100-105 (2009)

27. M Sikora, JN Laneman, M Haenggi, DJ Costello, TE Fuja, Bandwidth and power-efficient routing in linear wireless networks. IEEE Trans. Inf. Theory. 52(6), 2624-2633 (2006)

28. A Goldsmith, Wireless Communications. (Cambridge University Press, California, 2005)

29. DP Moya Osorio, EE Bentez Olivo, H Alves, JCS Santos Filho, M Latva-aho, Exploiting the direct link in full-duplex amplify-and-forward relaying networks. IEEE Signal Process. Lett. 22(10), 1766-1770 (2015)

30. GK Karagiannidis, NC Sagias, TA Tsiftsis, Closed-form statistics for the sum of squared Nakagami- $m$ variates and its applications. IEEE Trans. Commun. 54(8), 1353-1359 (2006)

31. FE Satterthwaite, An approximate distribution of estimates of variance components. Biom. Bull. 2(6), 110-114 (1946)

32. S Boyd, L Vandenberghe, Convex optimization. (Cambridge University Press, New York, 2004)

33. H Tuy, Convex analysis and global optimization. (Kluwer Academic, Hanoi, 1998)

34. HH Kha, HD Tuan, HH Nguyen, Fast global optimal power allocation in wireless networks by local d.c.programming. IEEE Trans. Wireless Commun. 11(2), 510-515 (2012)
35. A Beck, A Ben-Tal, L Tetruashvili, A sequential parametric convex approximation method with applications to non-convex truss topology design problems. J. Glob Optim. 47(1), 29-51 (2010)

36. M Grant, S Boyd, CVX: Matlab software for disciplined convex programming, version 1.21 (2010). Available: http://cvxr.com/cvx

37. S Boyd, SJ Kim, L Vandenberghe, A Hassibi, A tutorial on geometric programming. Optim. Eng. 8(1), 67-127 (2007)

38. D Mishra, S De, C-F Chiasserini, Joint optimization schemes for cooperative wireless information and power transfer over rician channels. IEEE Trans. Commun. 64(2), 554-571 (2016)

39. ZQ Luo, S Zhang, Dynamic spectrum management: complexity and duality. IEEE J. Sel. Top. Sign. Process. 2(1), 57-73 (2008)

40. IS Gradshteyn, IM Ryzhik, A Jeffrey, Table of Integrals, Series and Products, 6th edition. (Academic press, 2000)

\section{Submit your manuscript to a SpringerOpen ${ }^{\circ}$ journal and benefit from:}

- Convenient online submission

- Rigorous peer review

- Open access: articles freely available online

- High visibility within the field

- Retaining the copyright to your article

Submit your next manuscript at $\$$ springeropen.com 